\title{
4"'-N-Demethylspiramycin Derivatives: Synthesis and Evaluation of Effectiveness against Drug-resistant Bacteria
}

\author{
Toshiaki Sunazuka, Hiroko Shudo, Kenichiro Nagai, Kiminari Yoshida, \\ Yukie Yamaguchi, Hideaki Hanaki, Satoshi Ōmura
}

Received: February 14, 2008 / Accepted: March 11, 2008

(C) Japan Antibiotics Research Association

\begin{abstract}
Amino-4"-O-benzoyl-4"'- $N$-demethyl-18deoxospiramycins were designed and synthesized. Synthetic strategy involved selective demethylation of the dimethylamino group in forosamine, benzoylation of the hydroxyl group at the $\mathrm{C} 4$ " position and reductive $\mathrm{N}$ amination of the formyl group. Antibacterial characteristics of spiramycin derivatives were tested. The derivatives exhibited promising activity against drug-resistant bacterial strains.
\end{abstract}

Keywords spiramycin, MRSA, demethylation, reductive $\mathrm{N}$-amination

\section{Introduction}

Spiramycin I (1) is a 16-membered-ring macrolide with two aminosugars, mycaminose and forosamine together with one neutral sugar mycarose (Fig. 1). It shows antibacterial activities against Gram-positive bacteria and mycoplasmas [1]. Our group has continuously studied the synthesis and biological activity of spiramycin derivatives to elucidate their structure-activity relationships [2 7]. Recently, the spiramycin derivatives in our chemical library have been reinvestigated in the course of our screening for antibacterial agents. The spiramycin derivative 2a [4], one of byproducts in the silylacetal formation, was found to show

S. Ōmura (Corresponding author), T. Sunazuka, H. Shudo, K. Nagai, K. Yoshida, Y. Yamaguchi, H. Hanaki: Kitasato Institute for Life Sciences and Graduate School of Infection Control Sciences, Kitasato University and The Kitasato Institute, 5-9-1 Shirokane, Minato-ku, Tokyo 108-8641, Japan,

E-mail: omura-s@kitasato.or.jp moderate activity for drug-resistant bacteria, including methicillin-resistant Staphylococcus aureus (MRSA), whereas the parent compound $\mathbf{1}$ is inactive. Structurally, 2a has 3,18-( $O$-silyl)acetal, 4"-O-TBDMS and 4"'methylamino groups. However, the 3,18-(O-silyl)acetal and $4 "-O$-TBDMS groups are not appropriate for medicinal uses because of their lability under acid conditions. Nevertheless, we found that the TBDMS ether 2a can be converted to esters $\mathbf{2 b}$ and $\mathbf{2 c}$, which maintain activity against MRSA strains [8]. Moreover, acetylene compound $\mathbf{2 c}$ is useful for producing 1,2,3-triazole analogues via click chemistry [9]. If the remaining silylacetal moiety in $\mathbf{2}$ is converted to other functional groups without reducing activity, it could be a promising lead compound to combat drug-resistant bacteria. Herein, we report the design, synthesis and antibacterial activity of $4^{\prime \prime \prime}-\mathrm{N}$ demethylspiramycin derivatives $\mathbf{3}$ bearing various amines instead of the 3,18-(O-silyl)acetal moiety found in $\mathbf{2}$.

\section{Chemistry}

Synthesis of spiramycin derivatives $\mathbf{3}$ is summarized in Scheme 1. Selective $2^{\prime}$-acetylation of $\mathbf{1}$ with acetic anhydride yielded 2 '-O-acetylspiramycin 4 , which was converted to $4^{\prime \prime}$-benzoyl compound $\mathbf{5}$ in $72 \%$ yield through treatment with benzoic acid and DCC in the presence of catalytic DMAP. In this acylation step, the use of benzoic anhydride or benzoyl chloride as acylating agents gave lower yields of $\mathbf{5}$, accompanied by 3,4"-dibenzoyl derivative. Next, we attempted selective $N$-demethylation of the 4 "' -dimethylamino group in $\mathbf{5}$ using our method previously reported [6]. However, $N$-demethylation with NBS and $\mathrm{NaN}_{3}$ caused decomposition of $\mathbf{5}$. After several attempts, the reaction of $\mathbf{5}$ with $\mathrm{FmocCl}$ and $\mathrm{NaHCO}_{3}$ in EtOAc under reflux smoothly proceeded to provide $4{ }^{\prime \prime \prime}$ - 


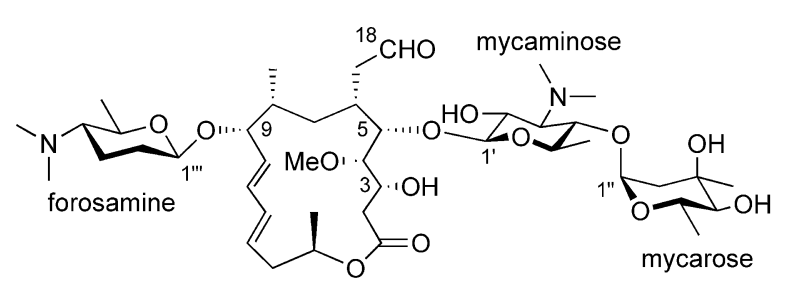

Spiramycin I (1)

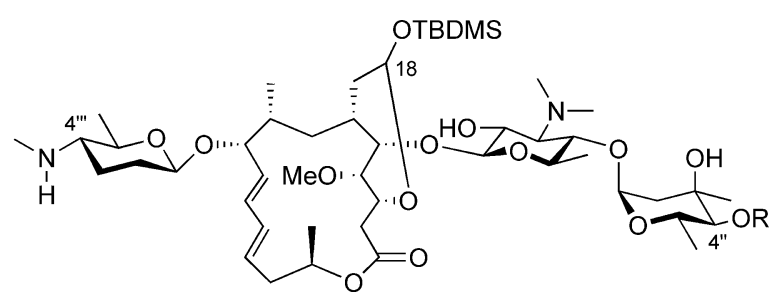

2a ( $R=$ TBDMS)

$2 b(R=B z)$

2c (R = 5-Hexynoyl)
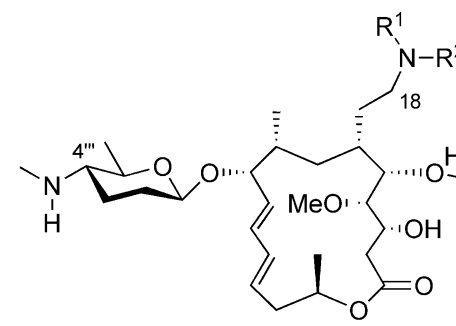

3

Fig. $\mathbf{1}$ Structures of spiramycin I (1), and compounds $\mathbf{2 a}, \mathbf{2} \mathbf{b}, \mathbf{2} \mathbf{c}$ and $\mathbf{3}$.

Fmoc protected spiramycin $\mathbf{6}$ in quantitative yield via $\mathrm{N}$ demethylation of the $4^{\prime \prime \prime}$-dimethylamino group without formation of $3^{\prime}$-Fmoc protected spiramycin. The selectivity of $4^{\prime \prime \prime}-N$-demethylation is due to less steric hindrance around the dimethylamino group of forosamine compared to that of mycaminose. It is noted that benzoylation of the 4 "-hydroxyl group did not work when 4 was protected with Fmoc group in advance. This result indicates that introduction of 4 "' $-N$-Fmoc group causes a conformational change that forosamine is close to mycarose, and then the Fmoc group shields around 4"-hydroxyl group in mycarose from attack of acylating agents. The formyl group of 6 was treated with a variety of amines in the presence of $\mathrm{NaBH}(\mathrm{OAc})_{3}$ and $\mathrm{AcOH}$ to afford amine compounds 7 in high yields [10]. Finally, deprotection of the Fmoc group with piperidine, followed by methanolysis of the acetyl group provided spiramycin derivatives $\mathbf{3}$ in good yields. DBU can be used for deprotection of the Fmoc group without decomposition of the spiramycin skeleton.

\section{Results and Discussion}

The in vitro antibacterial activities of spiramycin derivatives 3 were tested by the standard agar dilution method against $S$. aureus FDA209P (susceptible strain), S. aureus Smith (susceptible strain), MRSA HH-1 (methicillin-resistant S. aureus), MRSA HH-1 HR-1 (methicillin-resistant S. aureus), MRSA 70 (methicillinresistant S. aureus), MRSA 92-1191 (methicillin-resistant
S. aureus), S. aureus ISP447 (inducibly resistant strain), $S$. aureus ISP217 (constitutively resistant strain), S. aureus 8325 (pEP2104) (encoded by erm gene), S. aureus 8325 (pMS97) (encoded by erm and mef genes) shown in Table 1 .

Spiramycin I (1) displayed anitibacterial activity against $S$. aureus FDA209P and $S$. aureus Smith with an MIC value of 2.0 and $4.0 \mu \mathrm{g} / \mathrm{ml}$ respectively, but no activity for MRSA strains. Dibenzylamino spiramycin 3a was less active against EM susceptible strains compared to 1, while 3a showed antibacterial activity against MRSA strains with MIC values of $8.0 \sim 16 \mu \mathrm{g} / \mathrm{ml}$. However, 3a showed weak activity compared to vancomycin. Compound $\mathbf{3 b}$ was slightly less active than 3a. These results indicate that the replacement of the 3,18-( $O$-silyl)acetal moiety in $\mathbf{2 a}$ and $\mathbf{2 b}$ with a suitable amino group at $\mathrm{C} 18$ does not reduce activity significantly. Exceptionally, monobenzylamino spiramycin 3c lost activity against all tested strains. The phenyl group seems to have a crucial effect on antibacterial activity because pyridine derivatives $\mathbf{3 d}$ and $\mathbf{3 e}$ were inactive against all susceptible and resistant strains. The significance of the position and orientation of the phenyl group is emphasized because $\mathbf{3 c}$ and $\mathbf{3 f}$ having the phenyl group showed no activity. Furthermore, indoline $\mathbf{3 g}$ and tetrahydroquinoline $\mathbf{3 i}$ displayed moderate activity against MRSA strains, whereas isoindoline $\mathbf{3 h}$ and tetrahydroisoquinoline $\mathbf{3 j}$ diminished activity against all tested strains. To examine the effect of the three functional groups in $3 \mathrm{~g}$ on anti-MRSA activity, spiramycin derivatives $\mathbf{8}, 9$ and 10 were prepared (Fig. 2). Surprisingly, 8, 9 and 10 


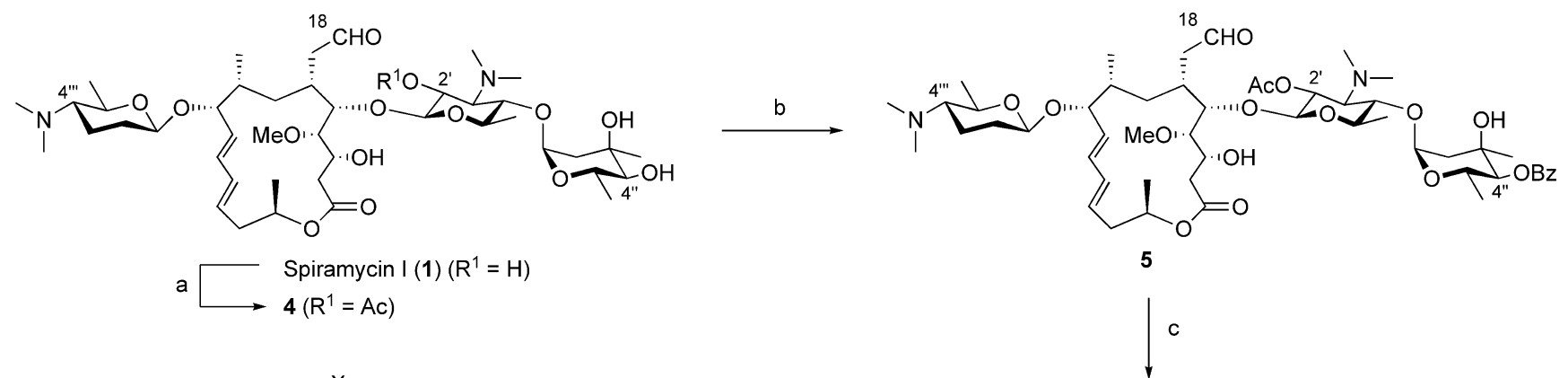

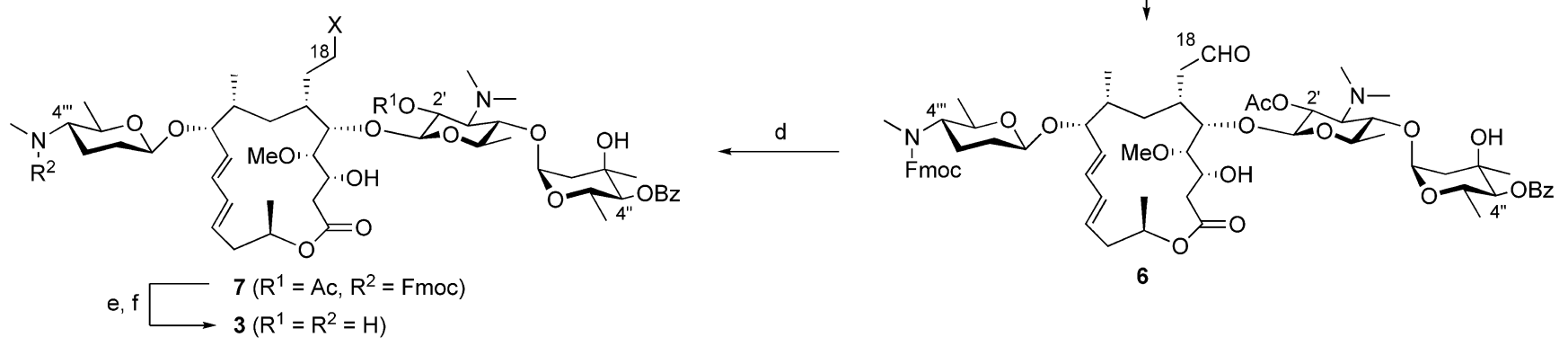

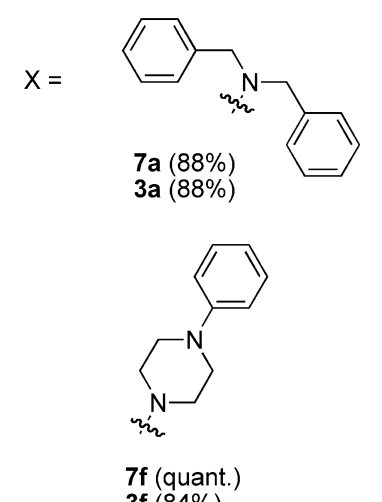

$3 f(84 \%)$

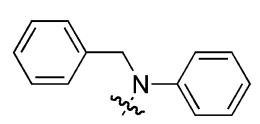

7b (quant.) 3b $(71 \%)$

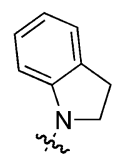

7g (quant.)

$7 g$ (quant.)
$\mathbf{3 g}(60 \%)$
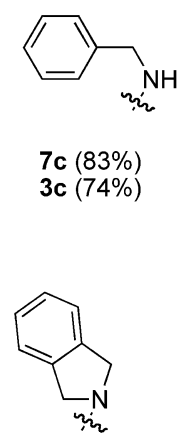

7h $(89 \%)$ 3h $(73 \%)$

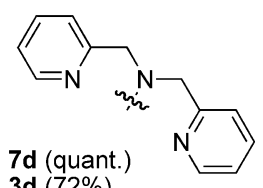

3d $(72 \%)$

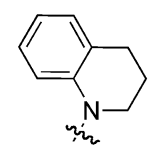

$7 \mathbf{i}(98 \%)$ $3 i$ (quant.)

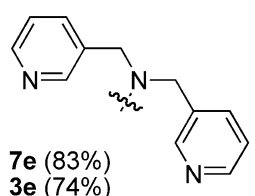

3e $(74 \%)$

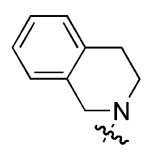

$7 j$ (98\%)

3j (quant.)

a) $\mathrm{Ac}_{2} \mathrm{O}$, acetone, rt, 5 hours, quant.; b) Benzoic acid, DCC, DMAP, $\mathrm{CH}_{2} \mathrm{Cl}_{2}, 0^{\circ} \mathrm{C} \sim \mathrm{rt}, 20$ hours, $72 \%$; c) $\mathrm{FmocCl}, \mathrm{NaHCO}_{3}, \mathrm{EtOAc}, \mathrm{reflux}$, 3.5 hours, quant.; d) amine, $\mathrm{AcOH}, \mathrm{NaBH}(\mathrm{OAc})_{3}, \mathrm{CHCl}_{3}, \mathrm{rt}, 81 \% \sim$ quant.; e) piperidine, EtOAc, rt or $\mathrm{DBU}, \mathrm{EtOAc}, \mathrm{rt}$; f) $\mathrm{MeOH}, \mathrm{reflux}$, $60 \% \sim$ quant. in 2 steps; . DCC $=N, N^{\prime}$-dicyclohexylcarbodiimide, DMAP=4-(dimethylamino)pyridine, Fmoc=9-Fluorenyl-methyloxycarbonyl.

Scheme 1 Synthesis of spiramycin derivatives 3.

showed no antibacterial activity, suggesting that combination of the $18-N$-indolino, 4"-O-benzoyl and $4^{\prime \prime \prime}-N$ methylamino groups bestows effectiveness against drugresistant strains.

\section{Conclusion}

In conclusion, we have demonstrated the synthesis and antibacterial activity of 18-amino-4"-O-benzoyl-4"'- $N$ demethyl-18-deoxospiramycins. The structural features of the potent compound, $\mathbf{3 g}$ include the $18-\mathrm{N}$-indolino, $4^{\prime \prime}-\mathrm{O}$ - benzoyl and 4"'-N-methylamino groups. Although $\mathbf{3 a}, \mathbf{3 b}$, $\mathbf{3 g}$ and $\mathbf{3 i}$ are less potent than vancomycin (MIC: MRSA strains; $0.5 \sim 1.0 \mu \mathrm{g} / \mathrm{ml}$ ), this result may offer useful information to help design 16-membered-ring macrolide derivatives with effectiveness against drug-resistant strains. Based on these results, further spiramycin derivatives are being investigated. The in vivo biological activity will be reported elsewhere. 
Table 1. In vitro antibacterial activities of spiramycin derivatives and vancomycin

\begin{tabular}{|c|c|c|c|c|c|c|c|c|c|c|c|c|c|c|c|}
\hline \multirow{2}{*}{ Strain/Compound } & \multicolumn{15}{|c|}{$\mathrm{MIC}(\mu \mathrm{g} / \mathrm{ml})$} \\
\hline & SPM & VCM & $2 a$ & $2 b$ & 2c & $3 a$ & $3 b$ & $3 c$ & $3 d$ & $3 e$ & $3 f$ & $3 \mathbf{g}$ & $3 h$ & $3 \mathbf{i}$ & $3 \mathbf{j}$ \\
\hline S. aureus FDA209P11) & 2 & 1 & 4 & 8 & 16 & 8 & 16 & 128 & 128 & $>64$ & 64 & 8 & $>64$ & 8 & 128 \\
\hline S. aureus Smith²) & 4 & 1 & 4 & 16 & 16 & 16 & - & $>128$ & $>128$ & $>64$ & 128 & 8 & $>64$ & 16 & $>128$ \\
\hline MRSA HH-13) & $>256$ & $\leqq 0.5$ & 4 & 8 & 16 & 8 & 8 & $>128$ & $>128$ & $>64$ & 64 & 4 & $>64$ & 8 & 128 \\
\hline MRSA HH-1 HR-1 ${ }^{4)}$ & $>256$ & $\leqq 0.5$ & 4 & 8 & 16 & 16 & 16 & $>128$ & $>128$ & $>64$ & 64 & 4 & $>64$ & 8 & 128 \\
\hline MRSA 705) & $>256$ & $\leqq 0.5$ & 4 & 8 & 16 & 8 & 16 & $>128$ & $>128$ & $>64$ & 64 & 64 & $>64$ & 8 & 128 \\
\hline MRSA 92-11916) & - & 1 & 4 & 16 & 32 & 16 & 32 & $>128$ & $>128$ & $>64$ & 128 & 4 & $>64$ & 8 & $>128$ \\
\hline S. aureus ISP4477) & 8 & 1 & 4 & 16 & 32 & 16 & 32 & $>128$ & $>128$ & $>64$ & 128 & 8 & $>64$ & 16 & $>128$ \\
\hline S. aureus ISP217 & $>256$ & $\leqq 0.5$ & 4 & 8 & 16 & 8 & 32 & $>128$ & $>128$ & $>64$ & 64 & 8 & $>64$ & 8 & 64 \\
\hline S. aureus $8325(\mathrm{pEP} 2104)^{9)}$ & 4 & 1 & $>64$ & 8 & 8 & $>128$ & $>64$ & $>128$ & 32 & $\leqq 0.5$ & 64 & 2 & $>128$ & 8 & 64 \\
\hline S. aureus 8325 (pMS97) ${ }^{10)}$ & 4 & 1 & $>64$ & 8 & 8 & $>128$ & $>64$ & $>128$ & 8 & 1 & 64 & 2 & 32 & 8 & 128 \\
\hline
\end{tabular}

1) Staphylococcus aureus FDA209P (susceptible strain), ${ }^{2)}$ S. aureus Smith (susceptible strain), ${ }^{3)} \mathrm{MRSA}$ HH-1 (methicillin-resistant S. aureus), ${ }^{4)}$ MRSA HH-1 HR-1 (methicillin-resistant S. aureus), ${ }^{5)}$ MRSA 70 (methicillin-resistant S. aureus), ${ }^{6)}$ MRSA 92-1191 (methicillin-resistant S. aureus), ${ }^{7)}$ S. aureus ISP447 (inducibly resistant strain), ${ }^{8)}$ S. aureus ISP217 (constitutively resistant strain), ${ }^{9)}$ S. aureus 8325 (pEP2104) (encoded by erm gene), 10) $S$. aureus 8325 (pMS97) (encoded by erm and mef genes).

SPM: spiramycin; VCM: vancomycin.

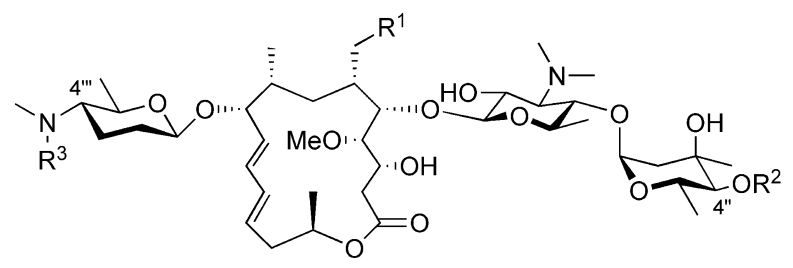

\begin{tabular}{cccc}
\hline & $\mathrm{R}^{1}$ & $\mathrm{R}^{2}$ & $\mathrm{R}^{3}$ \\
\hline $\mathbf{8}$ & $\mathrm{CH}_{2}-\mathrm{N}$-indoline & $\mathrm{H}$ & $\mathrm{H}$ \\
$\mathbf{9}$ & $\mathrm{CH}_{2}-\mathrm{N}$-indoline & $\mathrm{Bz}$ & $\mathrm{Me}$ \\
$\mathbf{1 0}$ & $\mathrm{CHO}$ & $\mathrm{Bz}$ & $\mathrm{H}$ \\
\hline
\end{tabular}

Fig. 2 Structures of spiramycin derivatives $\mathbf{8}, \mathbf{9}$ and $\mathbf{1 0 .}$

\section{Experimental}

Reagents were purchased at highest commercial quality and used without further purification, unless specified otherwise. Reactions were monitored by TLC using Merck $\mathrm{F} 60_{254}$ silica gel plates. The plates were visualized with UV light and stained with phosphomolybdic acid. Silica gel chromatography was performed on Merck Kieselgel 60 (Art. 1.09385).

FT-IR spectra were recorded on a Horiba FT-210 spectrometer. Mass spectra were recorded on a JEOL JMSAX505 HA Mass Spectrometer. ${ }^{1} \mathrm{H}-\mathrm{NMR}$ spectra were recorded on a JEOL JNM-EX270 spectrometer in $\mathrm{CDCl}_{3}$, $\mathrm{CD}_{3} \mathrm{OD}$ or pyridine- $d_{5} .{ }^{1} \mathrm{H}-\mathrm{NMR}$ spectral data are reported as follows: chemical shifts relative to chloroform (7.26 $\mathrm{ppm})$, multiplicity $(\mathrm{s}=$ singlet, $\mathrm{d}=$ doublet, $\mathrm{t}=$ triplet, $\mathrm{q}=$ quartet, $\mathrm{m}=$ multiplet, $\mathrm{br}=$ broad), coupling and integration.

\section{2'-O-Acetylspiramycin I (4)}

To a solution of spiramycin I (1) $(6.70 \mathrm{~g}, 7.95 \mathrm{mmol})$ in $\mathrm{Me}_{2} \mathrm{CO}(9.9 \mathrm{ml})$ was added acetic anhydride $(2.25 \mathrm{ml}$, $23.8 \mathrm{mmol}$ ), and the mixture was stirred at room temperature. After stirring for 5 hours, the solvent was concentrated under reduced pressure. The residue was diluted with $\mathrm{CHCl}_{3}$ and washed with saturated aq $\mathrm{NaHCO}_{3}$ and $\mathrm{H}_{2} \mathrm{O}$. The organic layer was dried over anhydrous $\mathrm{Na}_{2} \mathrm{SO}_{4}$, filtered and evaporated to give 4 (7.03 g, quant.) as a colorless powder.

HR-MS (FAB) (matrix; $m$-NBA): calcd. for $\mathrm{C}_{45} \mathrm{H}_{77} \mathrm{~N}_{2} \mathrm{O}_{15}: 885.5324[\mathrm{M}+\mathrm{H}]$, found: $m / z 885.5328$ $[\mathrm{M}+\mathrm{H}]^{+}$.

IR $v_{\max }(\mathrm{KBr}) \mathrm{cm}^{-1}: 3466,2935,1726$.

${ }^{1} \mathrm{H}-\mathrm{NMR}\left(270 \mathrm{MHz}, \mathrm{CDCl}_{3}\right.$, partial data) $\delta$ (ppm): 9.73 (s, 1H, H-18), 6.17 (dd, $J=14.9,10.5 \mathrm{~Hz}, 1 \mathrm{H}, \mathrm{H}-11$ ), 5.91 
(dd, $J=14.9,9.7 \mathrm{~Hz}, 1 \mathrm{H}, \mathrm{H}-12), 5.61$ (dd, $J=15.1,9.5 \mathrm{~Hz}$ $1 \mathrm{H}, \mathrm{H}-10$ ), 5.48 (ddd, $J=15.7,13.5,2.7 \mathrm{~Hz}, 1 \mathrm{H}, \mathrm{H}-13$ ), 5.21 (m, 1H, H-15), 4.98 (d, J=3.0 Hz, 1H, H-1"), 4.90 (dd, $\left.J=10.3,7.6 \mathrm{~Hz}, 1 \mathrm{H}, \mathrm{H}-2^{\prime}\right), 4.60$ (d, $\left.J=7.8 \mathrm{~Hz}, 1 \mathrm{H}, \mathrm{H}-1^{\prime}\right)$, 4.30 (d, $J=8.4 \mathrm{~Hz}, 1 \mathrm{H}, \mathrm{H}-1^{\prime \prime \prime}$ ), $4.05 \sim 3.94$ (m, 3H, H-5, H9, H-5"), 3.71 (d, $J=11.3 \mathrm{~Hz}, 1 \mathrm{H}, \mathrm{H}-3), 3.38$ (s, 3H, 4$\left.\mathrm{OCH}_{3}\right), 3.34\left(\mathrm{~m}, 1 \mathrm{H}, \mathrm{H}-5^{\prime \prime \prime}\right), 3.23 \sim 3.21\left(\mathrm{~m}, 2 \mathrm{H}, \mathrm{H}-4^{\prime}, \mathrm{H}-\right.$ $\left.5^{\prime}\right), 2.92$ (d, $\left.J=8.9 \mathrm{~Hz}, 1 \mathrm{H}, \mathrm{H}-4\right), 2.86$ (d, $J=10.0 \mathrm{~Hz}, 1 \mathrm{H}$, $\left.\mathrm{H}-4^{\prime \prime}\right), 2.32$ (s, 3H, 3'-N(CH$\left.)_{2}\right), 2.30\left(\mathrm{~s}, 3 \mathrm{H}, 3^{\prime}-\mathrm{N}\left(\mathrm{CH}_{3}\right)_{2}\right)$, $2.18\left(\mathrm{~s}, 6 \mathrm{H}, 4^{\prime \prime \prime}-\mathrm{N}\left(\mathrm{CH}_{3}\right)_{2}\right), 1.96\left(\mathrm{~s}, 3 \mathrm{H}, 2^{\prime}-\mathrm{OCOCH}_{3}\right), 1.23$ (d, $J=6.5 \mathrm{~Hz}, 3 \mathrm{H}), 1.21$ (d, $J=6.5 \mathrm{~Hz}, 3 \mathrm{H}), 1.16(\mathrm{~m}, 15 \mathrm{H}$, $\left.15-\mathrm{CH}_{3}, 3^{\prime \prime}-\mathrm{CH}_{3}, 5^{\prime}-\mathrm{CH}_{3}, 5^{\prime \prime}-\mathrm{CH}_{3}, 5^{\prime \prime \prime}-\mathrm{CH}_{3}\right), 0.89$ (d, $J=6.5$ $\left.\mathrm{Hz}, 3 \mathrm{H}, 8-\mathrm{CH}_{3}\right), 0.81$ (m, $\left.1 \mathrm{H}, \mathrm{H}-7\right)$.

\section{2'-O-Acetyl-4"-O-benzoylspiramycin I (5)}

To a solution of $4(3.03 \mathrm{~g}, 3.42 \mathrm{mmol})$ in $\mathrm{CH}_{2} \mathrm{Cl}_{2}(34 \mathrm{ml})$ was added benzoic acid $(2.09 \mathrm{~g}, 17.0 \mathrm{mmol}$, DMAP (207 $\mathrm{mg}, 1.70 \mathrm{mmol}$ and $\mathrm{DCC}\left(3.51 \mathrm{~g}, 17.0 \mathrm{mmol}\right.$ at $0^{\circ} \mathrm{C}$ under $\mathrm{N}_{2}$. After stirring for 1 hour, the mixture was warmed to room temperature and further stirred for 19 hours. The mixture was cooled to $0^{\circ} \mathrm{C}$ and then saturated aq $\mathrm{NH}_{4} \mathrm{Cl}$ was added. The resulting mixture was extracted with EtOAc, washed with saturated aq $\mathrm{NaHCO}_{3}$ and brine, dried over anhydrous $\mathrm{Na}_{2} \mathrm{SO}_{4}$, filtered and evaporated. The residue was purified by column chromatography on silica gel with an eluent $\left(\mathrm{CH}_{2} \mathrm{Cl}_{2}: \mathrm{MeOH}: \mathrm{NH}_{4} \mathrm{OH}=80: 1: 0.1\right.$ to $60: 1: 0.1)$ to give $5(2.43 \mathrm{~g}, 72 \%)$ as a colorless powder.

HR-MS (FAB) (matrix; $m$-NBA): calcd. for $\mathrm{C}_{52} \mathrm{H}_{81} \mathrm{~N}_{2} \mathrm{O}_{16}: 989.5586[\mathrm{M}+\mathrm{H}]$, found: $m / z 989.5598$ $[\mathrm{M}+\mathrm{H}]^{+}$.

IR $v_{\max }(\mathrm{KBr}) \mathrm{cm}^{-1}: 3505,2975,2935,2875,2842$, 1749, 1724.

${ }^{1} \mathrm{H}-\mathrm{NMR}\left(270 \mathrm{MHz}, \mathrm{CDCl}_{3}\right.$, partial data) $\delta$ (ppm): 9.80 (s, 1H, H-18), 8.11 8.04 (m, 2H, 4"-OCOPh), 7.55 (m, 1H, 4"-OCOPh), 7.49 7.39 (m, 2H, 4"-OCOPh), 6.23 (dd, $J=16.2,10.5 \mathrm{~Hz}, 1 \mathrm{H}, \mathrm{H}-11), 5.96$ (dd, $J=14.4,10.5 \mathrm{~Hz}$, 1H, H-12), 5.65 (dd, $J=15.1,9.6 \mathrm{~Hz}, 1 \mathrm{H}, \mathrm{H}-10$ ), 5.53 (ddd, $J=15.0,11.0,3.9 \mathrm{~Hz}, 1 \mathrm{H}, \mathrm{H}-13), 5.27$ (m, 1H, H-15), 5.11 (d, $\left.J=3.2 \mathrm{~Hz}, 1 \mathrm{H}, \mathrm{H}-1^{\prime \prime}\right), 4.98$ (dd, $J=10.4,7.6 \mathrm{~Hz}, 1 \mathrm{H}, \mathrm{H}-$ $2^{\prime}$ ), 4.81 (d, $\left.J=10.0 \mathrm{~Hz}, 1 \mathrm{H}, \mathrm{H}-4^{\prime \prime}\right), 4.70$ (d, $J=7.6 \mathrm{~Hz}, 1 \mathrm{H}$, H-1'), 4.51 (dq, $\left.J=10.1,6.5 \mathrm{~Hz}, 1 \mathrm{H}, \mathrm{H}-5^{\prime \prime}\right), 4.34$ (d, $J=7.8$ $\left.\mathrm{Hz}, 1 \mathrm{H}, \mathrm{H}-1^{\prime \prime \prime}\right), 4.13 \sim 4.01$ (m, 2H, H-5, H-9), 3.78 (d, $J=11.3 \mathrm{~Hz}, 1 \mathrm{H}, \mathrm{H}-3), 3.45$ (s, $\left.3 \mathrm{H}, 4-\mathrm{OCH}_{3}\right), 3.40$ (dq, $\left.J=9.5,6.2 \mathrm{~Hz}, 1 \mathrm{H}, \mathrm{H}-5^{\prime \prime \prime}\right), 3.34 \sim 3.31$ (m, 2H, H-4', H-5'), 2.98 (d, $J=9.0 \mathrm{~Hz}, 1 \mathrm{H}, \mathrm{H}-4), 2.44$ (s, 6H, 3'-N $\left.\left(\mathrm{CH}_{3}\right)_{2}\right), 2.25$ (s, 6H, 4"'-- $\left.\left(\mathrm{CH}_{3}\right)_{2}\right), 2.04$ (s, 3H, 2'-OCOCH$\left.H_{3}\right), 1.29$ (d, $\left.J=6.5 \mathrm{~Hz}, 3 \mathrm{H}, 15-\mathrm{CH}_{3}\right), 1.23\left(\mathrm{~d}, J=6.5 \mathrm{~Hz}, 3 \mathrm{H}, 5^{\prime}-\mathrm{CH}_{3}\right.$ ), $1.18\left(\mathrm{~d}, J=5.1 \mathrm{~Hz}, 3 \mathrm{H}, \mathrm{C}^{\prime \prime}-\mathrm{CH}_{3}\right), 1.14(\mathrm{~d}, J=6.5 \mathrm{~Hz}, 3 \mathrm{H}$, 5"'-C $\left.H_{3}\right), 1.18$ (s, 3H, 3"-CH $), 0.95$ (d, $J=6.5 \mathrm{~Hz}, 3 \mathrm{H}, 8-$ $\left.\mathrm{CH}_{3}\right), 0.87$ (m, 1H, H-7).
2' -O-Acetyl-4"-O-benzoyl-4"'- $N$-demethyl-4"'- $N$-(9fluorenylmethoxycarbonyl)spiramycin I (6)

To a solution of 5 (3.13 g, $3.17 \mathrm{mmol})$ in EtOAc (32 ml) were added $\mathrm{NaHCO}_{3}(1.34 \mathrm{~g}, 17.0 \mathrm{mmol})$ and 9fluorenylmethylchloroformate $(2.85 \mathrm{~g}, 11.0 \mathrm{mmol})$, and the mixture was stirred under reflux. After stirring for 3.5 hours, the mixture was cooled to room temperature. The mixture was diluted with EtOAc and washed with water and brine, dried over anhydrous $\mathrm{Na}_{2} \mathrm{SO}_{4}$, filtered and evaporated. The residue was purified by column chromatography on silica gel with an eluent (hexane: $\mathrm{EtOAc}=3: 1$ to $1: 1)$ to give $6(3.80 \mathrm{~g}$, quant.) as a colorless powder.

HR-MS (FAB) (matrix; $m$-NBA): calcd. for $\mathrm{C}_{66} \mathrm{H}_{89} \mathrm{~N}_{2} \mathrm{O}_{18}: 1197.6110[\mathrm{M}+\mathrm{H}]$, found: $m / z 1197.6152$ $[\mathrm{M}+\mathrm{H}]^{+}$.

IR $v_{\max }(\mathrm{KBr}) \mathrm{cm}^{-1}: 3500,2975,2935,2842,1749$, 1722,1704 .

${ }^{1} \mathrm{H}-\mathrm{NMR}\left(270 \mathrm{MHz}, \mathrm{CDCl}_{3}\right.$, partial data) $\delta$ (ppm): 9.80 (s, 1H, H-18), 8.08 (d, J=7.3 Hz, 2H, 4"-OCOPh), 7.76 (d, $J=7.3 \mathrm{~Hz}, 2 \mathrm{H}$, Fmoc), $7.56 \sim 7.52$ (m, 3H, 4"-OCOPh), $7.45 \sim 7.28$ (m, 6H, Fmoc), 6.23 (dd, $J=15.1,10.5 \mathrm{~Hz}, 1 \mathrm{H}$, H-11), 5.97 (dd, $J=14.9,10.5 \mathrm{~Hz}, 1 \mathrm{H}, \mathrm{H}-12), 5.65$ (dd, $J=15.1,10.0 \mathrm{~Hz}, 1 \mathrm{H}, \mathrm{H}-10), 5.54(\mathrm{~m}, 1 \mathrm{H}, \mathrm{H}-13), 5.27$ (m, 1H, H-15), 5.11 (d, J=3.0 Hz, 1H, H-1"), 4.97 (m, 1H, H$\left.2^{\prime}\right), 4.83$ (d, J=10.3 Hz, 1H, H-4"), 4.69 (m 1H, H-1'), 4.53 (m 1H, H-5"), 4.42 4.25 (m, 3H, H-1"', Fmoc), 4.23 4.00 (m, 3H, H-5, H-9, Fmoc), 3.79 (d, $J=11.0 \mathrm{~Hz}, 1 \mathrm{H}, \mathrm{H}-3$ ), 3.59 (m 1H, H-5"'), 3.47 (m, 3H, 4-OCH $_{3}$, 3.31 3.30 (m, 2H, H-4', H-5'), 2.98 (d, J=8.9 Hz, 1H, H-4), 2.72 (br.s, $\left.3 \mathrm{H}, 4^{\prime \prime \prime}-\mathrm{NCH}_{3}\right), 2.43\left(\mathrm{~s}, 6 \mathrm{H}, 3^{\prime}-\mathrm{N}\left(\mathrm{CH}_{3}\right)_{2}\right), 2.03$ (s, 3H, 2'$\left.\mathrm{OCOCH}_{3}\right), 1.29$ (d, $\left.J=6.5 \mathrm{~Hz}, 3 \mathrm{H}, 15-\mathrm{CH}_{3}\right), 1.18-1.14$ (m, $\left.9 \mathrm{H}, 5^{\prime}-\mathrm{CH}_{3}, 3^{\prime \prime}-\mathrm{CH}_{3}, 5^{\prime \prime}-\mathrm{CH}_{3}\right), 1.08$ (d, $J=6.5 \mathrm{~Hz}, 3 \mathrm{H}, 5^{\prime \prime \prime}-$ $\left.\mathrm{CH}_{3}\right), 0.96\left(\mathrm{~d}, J=6.5 \mathrm{~Hz}, 3 \mathrm{H}, 8-\mathrm{CH}_{3}\right), 0.86(\mathrm{~m}, 1 \mathrm{H}, \mathrm{H}-7)$.

\section{2'-O-Acetyl-4"-O-benzoyl-4"'- $N$-demethyl-18-( $N, N-$ dibenzyl)amino-4"'-N-(9-fluorenylmethoxycarbonyl)-18- deoxospiramycin I (7a)}

To a solution of $\mathbf{6}(78.5 \mathrm{mg}, 65.6 \mu \mathrm{mol})$ in $\mathrm{CHCl}_{3}(656 \mu \mathrm{l})$ were added $N, N$-dibenzylamine $(19.0 \mu 1,98.4 \mu \mathrm{mol})$ and $\mathrm{AcOH}(9.40 \mu \mathrm{l}, 164 \mu \mathrm{mol})$, and the mixture was stirred at room temperature for 15 minutes. Then $\mathrm{NaHB}(\mathrm{OAc})_{3}(21.0$ $\mathrm{mg}, 98.4 \mu \mathrm{mol}$ ) was added, and stirring continued for 30 minutes. The mixture was diluted with $\mathrm{CHCl}_{3}$ and washed with saturated aq $\mathrm{NaHCO}_{3}$ and brine, dried over anhydrous $\mathrm{Na}_{2} \mathrm{SO}_{4}$, filtered and evaporated. The residue was purified by column chromatography on silica gel with an eluent (hexane $: \mathrm{EtOAc}=3: 1$ to $1: 1$ ) to give $7 \mathbf{a}(79.3 \mathrm{mg}, 88 \%$ ) as a colorless powder.

HR-MS (FAB) (matrix; $m$-NBA): calcd. for $\mathrm{C}_{80} \mathrm{H}_{104} \mathrm{~N}_{3} \mathrm{O}_{17}: 1378.7366$ [M+H], found: $m / z 1378.7379$ 
$[\mathrm{M}+\mathrm{H}]^{+}$.

IR $v_{\max }(\mathrm{KBr}) \mathrm{cm}^{-1}: 3668,2975,2935,2842,1749$, 1722, 1704.

${ }^{1} \mathrm{H}-\mathrm{NMR}\left(270 \mathrm{MHz}, \mathrm{CDCl}_{3}\right.$, partial data) $\delta$ (ppm): 8.10 (d, $\left.J=7.3 \mathrm{~Hz}, 2 \mathrm{H}, 4^{\prime \prime}-\mathrm{OCO} P h\right), 7.75(\mathrm{~d}, J=7.3 \mathrm{~Hz}, 2 \mathrm{H}$, Fmoc), $7.59 \sim 7.54$ (m, 3H, 4"-OCOPh), 7.47 7.23 (m, $\left.16 \mathrm{H}, 18-\mathrm{N}-\left(\mathrm{CH}_{2} P h\right)_{2}, \mathrm{Fmoc}\right), 6.01 \sim 5.96(\mathrm{~m}, 2 \mathrm{H}, \mathrm{H}-11, \mathrm{H}-$ 12), 5.65 (dd, $J=15.1,10.0 \mathrm{~Hz}, 1 \mathrm{H}, \mathrm{H}-10), 5.57$ (m, $1 \mathrm{H}, \mathrm{H}-$ 13), 5.33 (m, 1H, H-15), 5.09 (d, $\left.J=3.2 \mathrm{~Hz}, 1 \mathrm{H}, \mathrm{H}-1^{\prime \prime}\right), 4.99$ (m, 1H, H-2') 4.83 (d, J=10.3 Hz, 1H, H-4"), 4.74 (d, $J=7.3 \mathrm{~Hz}, 1 \mathrm{H}, \mathrm{H}-1^{\prime}$ ), 4.54 (m 1H, H-5"), 4.43 4.38 (m, $3 \mathrm{H}, \mathrm{H}-1^{\prime \prime \prime}$, Fmoc), 4.23 4.07 (m, 3H, H-5, H-9, Fmoc), 3.47 (s, 3H, 4-OCH $H_{3}$ ), $3.29 \sim 3.26$ (m, 2H, H-4', H-5'), 3.01 (d, $J=8.6 \mathrm{~Hz}, 1 \mathrm{H}, \mathrm{H}-4), 2.63$ (br s, 3H, 4"'-- $\mathrm{NCH}_{3}$ ), $2.44\left(\mathrm{~s}, 6 \mathrm{H}, 3^{\prime}-\mathrm{N}\left(\mathrm{CH}_{3}\right)_{2}\right), 2.03\left(\mathrm{~s}, 3 \mathrm{H}, 2^{\prime}-\mathrm{OCOCH}_{3}\right), 1.30$ (d, $\left.J=6.5 \mathrm{~Hz}, 3 \mathrm{H}, 15-\mathrm{CH}_{3}\right), 1.20 \sim 1.17\left(\mathrm{~m}, 9 \mathrm{H}, 5^{\prime}-\mathrm{CH}_{3}, 5^{\prime \prime}-\right.$ $\mathrm{CH}_{3}, 3^{\prime \prime}-\mathrm{CH}_{3}$ ), 1.08 (d, $\left.\mathrm{J}=6.5 \mathrm{~Hz}, 3 \mathrm{H}, 5^{\prime \prime \prime}-\mathrm{CH}_{3}\right), 0.96$ (d, $\left.J=6.5 \mathrm{~Hz}, 3 \mathrm{H}, 8-\mathrm{CH}_{3}\right), 0.81$ (m, $\left.1 \mathrm{H}, \mathrm{H}-7\right)$.

\section{2'-O-Acetyl-4"'-O-benzoyl-4"'- $N$-demethyl-18-( $N, N$ - dibenzyl)amino-18-deoxospiramycin I}

To a solution of $7 \mathbf{a}(70.8 \mathrm{mg}, 51.4 \mu \mathrm{mol})$ in EtOAc $(514 \mu \mathrm{l})$ was added piperidine $(5.60 \mu \mathrm{l}, 56.5 \mu \mathrm{mol})$ and the mixture was stirred at room temperature. After stirring for 6 hours, the mixture was diluted with a $1: 1$ mixture of EtOAc and hexane, washed with saturated aq $\mathrm{NaHCO}_{3}$ and brine, dried over anhydrous $\mathrm{Na}_{2} \mathrm{SO}_{4}$, filtered and evaporated. The residue was purified by column chromatography on silica gel with an eluent $\left(\mathrm{CHCl}_{3}: \mathrm{MeOH}: \mathrm{NH}_{4} \mathrm{OH}=50: 1: 0.1\right)$ to give the title compound $(62.0 \mathrm{mg}$, quant.) as a colorless powder.

HR-MS (FAB) (matrix; $m$-NBA): calcd. for $\mathrm{C}_{65} \mathrm{H}_{94} \mathrm{~N}_{3} \mathrm{O}_{15}: 1156.6685[\mathrm{M}+\mathrm{H}]$, found: $m / z 1156.6741$ $[\mathrm{M}+\mathrm{H}]^{+}$.

${ }^{1} \mathrm{H}-\mathrm{NMR}\left(270 \mathrm{MHz}, \mathrm{CDCl}_{3}\right.$, partial data) $\delta$ (ppm): 8.09 (d, $\left.J=7.0 \mathrm{~Hz}, 2 \mathrm{H}, 4^{\prime \prime}-\mathrm{OCO} P h\right), 7.57$ (m, 1H, 4"-OCOPh), $7.47 \sim 7.22$ (m, 12H, 4"-OCOPh, 18- $\left.N-\left(\mathrm{CH}_{2} P h\right)_{2}\right), 6.02 \sim$ 5.94 (m, 2H, H-11, H-12), 5.63 (dd, $J=14.6,9.7 \mathrm{~Hz}, 1 \mathrm{H}, \mathrm{H}-$ 10), 5.52 (ddd, $J=14.3,10.5,3.8 \mathrm{~Hz}, 1 \mathrm{H}, \mathrm{H}-13$ ), 5.31 (m, $1 \mathrm{H}, \mathrm{H}-15$ ), 5.08 (d, $\left.J=3.5 \mathrm{~Hz}, 1 \mathrm{H}, \mathrm{H}-1^{\prime \prime}\right), 4.97$ (dd, $J=10.4$, $\left.7.8 \mathrm{~Hz}, 1 \mathrm{H}, \mathrm{H}-2^{\prime}\right), 4.82$ (d, $\left.J=10.3 \mathrm{~Hz}, 1 \mathrm{H}, \mathrm{H}-4^{\prime \prime}\right), 4.72$ (d, $\left.J=7.6 \mathrm{~Hz}, 1 \mathrm{H}, \mathrm{H}-1^{\prime}\right), 4.53$ (m 1H, H-5'), 4.25 (dd, $J=9.5$, $\left.1.9 \mathrm{~Hz}, 1 \mathrm{H}, \mathrm{H}-1^{\prime \prime \prime}\right), 4.06$ (d, J=8.9 Hz, 1H, H-5), 3.92 (m, 1H, H-9), 3.74 (d, $J=11.6 \mathrm{~Hz}, 1 \mathrm{H}, \mathrm{H}-3$ ), 3.66 (br s, 2H, 18$\left.N-\left(\mathrm{CH}_{2} P h\right)_{2}\right), 3.46$ (s, 3H, 4-OCH $\left.{ }_{3}\right), 3.40$ (br s, $2 \mathrm{H}, 18-\mathrm{N}-$ $\left.\left(\mathrm{CH}_{2} P h\right)_{2}\right), 3.29 \sim 3.25$ (m, 2H, H-4', H-5'), 3.01 (m, 1H, H-5"'), 2.97 (d, $J=9.7 \mathrm{~Hz}, 1 \mathrm{H}, \mathrm{H}-4), 2.42$ (s, 6H, 3'$\left.\mathrm{N}\left(\mathrm{CH}_{3}\right)_{2}\right), 2.39\left(\mathrm{~s}, 3 \mathrm{H}, 4^{\prime \prime \prime}-\mathrm{NHCH}_{3}\right), 2.07\left(\mathrm{~s}, 3 \mathrm{H}, 2^{\prime}-\right.$ $\left.\mathrm{OCOCH}_{3}\right), 1.30\left(\mathrm{~d}, J=6.5 \mathrm{~Hz}, 3 \mathrm{H}, 15-\mathrm{CH}_{3}\right), 1.17$ (m, 9H, $\left.5^{\prime}-\mathrm{CH}_{3}, 3^{\prime \prime}-\mathrm{CH}_{3}, 5^{\prime \prime}-\mathrm{CH}_{3}\right), 1.05$ (d, $\left.J=6.5 \mathrm{~Hz}, 3 \mathrm{H}, 5^{\prime \prime \prime}-\mathrm{CH}_{3}\right)$, 0.95 (d, $\left.J=6.5 \mathrm{~Hz}, 3 \mathrm{H}, 8-\mathrm{CH}_{3}\right), 0.77$ (m, 1H, H-7).
4"-O-Benzoyl-4"'-N-demethyl-18-( $N, N$-dibenzyl)amino18-deoxospiramycin I (3a)

A solution of $2^{\prime}-O$-acetyl compound $(55.2 \mathrm{mg}, 48.0 \mu \mathrm{mol})$ in $\mathrm{MeOH}(480 \mu \mathrm{l})$ was refluxed. After stirring for 12 hours, the solvent was removed. The residue was purified by column chromatography on silica gel with an eluent $\left(\mathrm{CHCl}_{3}: \mathrm{MeOH}: \mathrm{NH}_{4} \mathrm{OH}=60: 1: 0.1 \sim 20: 1: 0.1\right)$ to give 3a $(46.9 \mathrm{mg}, 88 \%)$ as a colorless powder.

HR-MS (FAB) (matrix; $m$-NBA): calcd. for $\mathrm{C}_{63} \mathrm{H}_{92} \mathrm{~N}_{3} \mathrm{O}_{14}: 1114.6579[\mathrm{M}+\mathrm{H}]$, found: $m / z 1114.6595$ $[\mathrm{M}+\mathrm{H}]^{+}$.

IR $v_{\max }(\mathrm{KBr}) \mathrm{cm}^{-1}: 3496,2973,2933,1722$.

${ }^{1} \mathrm{H}-\mathrm{NMR}\left(270 \mathrm{MHz}, \mathrm{CDCl}_{3}\right.$, partial data) $\delta(\mathrm{ppm}): 8.11$ (d, $\left.J=7.0 \mathrm{~Hz}, 2 \mathrm{H}, 4^{\prime \prime}-\mathrm{OCO} P h\right), 7.57$ (m, 1H, 4"-OCOPh), $7.48 \sim 7.26\left(\mathrm{~m}, \quad 12 \mathrm{H}, \quad 18-\mathrm{N}-\left(\mathrm{CH}_{2} \mathrm{Ph}\right)_{2}, \quad 4 "-\mathrm{OCO} P h\right)$, 5.99 5.94 (m, 2H, H-11, H-12), 5.61 (m, 1H, H-10), 5.52 (m, 1H, H-13), 5.33 (m, 1H, H-15), 5.10 (d, J=3.0 Hz, 1H, H-1"), 4.83 (d, $\left.J=10.3 \mathrm{~Hz}, 1 \mathrm{H}, \mathrm{H}-4^{\prime \prime}\right), 4.62$ (m 1H, H-5"), $4.31\left(\mathrm{~d}, J=8.1 \mathrm{~Hz}, 1 \mathrm{H}, \mathrm{H}-1^{\prime}\right), 4.04 \sim 4.01(\mathrm{~m}, 2 \mathrm{H}, \mathrm{H}-5, \mathrm{H}-$ $\left.1^{\prime \prime \prime}\right), 3.94(\mathrm{~m}, 1 \mathrm{H}, \mathrm{H}-9), 3.76 \sim 3.71(\mathrm{~m}, 3 \mathrm{H}, \mathrm{H}-3,18-\mathrm{N}$ $\left.\left(\mathrm{CH}_{2} \mathrm{Ph}\right)_{2}\right), 3.52\left(\mathrm{~m}, 1 \mathrm{H}, \mathrm{H}-2^{\prime}\right), 3.50\left(\mathrm{~s}, 3 \mathrm{H}, 4-\mathrm{OCH}_{3}\right), 3.36$ (d, $\left.J=14.0 \mathrm{~Hz}, 2 \mathrm{H}, 18-\mathrm{N}-\left(\mathrm{CH}_{2} P h\right)_{2}\right), 2.51\left(\mathrm{~s}, 6 \mathrm{H}, 3^{\prime}-\right.$ $\left.\mathrm{N}\left(\mathrm{CH}_{3}\right)_{2}\right), 2.42$ (s, 3H, 4"'--NHCH$\left.{ }_{3}\right), 1.31$ (d, $J=6.5 \mathrm{~Hz}, 3 \mathrm{H}$, $\left.15-\mathrm{CH}_{3}\right), 1.17\left(\mathrm{~m}, 12 \mathrm{H}, 5^{\prime}-\mathrm{CH}_{3}, 3^{\prime \prime}-\mathrm{CH}_{3}, 5^{\prime \prime}-\mathrm{CH}_{3}, 5^{\prime \prime \prime}-\mathrm{CH}_{3}\right)$, 0.94 (d, $\left.J=6.5 \mathrm{~Hz}, 3 \mathrm{H}, 8-\mathrm{CH}_{3}\right), 0.87$ (m, 1H, H-7).

\section{4"-O-Benzoyl-18-( $N$-benzyl- $N$-phenyl)amino-4"'- $N$ - demethyl-18-deoxospiramycin I (3b)}

HR-MS (FAB) (matrix; $m$-NBA): calcd. for $\mathrm{C}_{62} \mathrm{H}_{90} \mathrm{~N}_{3} \mathrm{O}_{14}$ : $1100.6423[\mathrm{M}+\mathrm{H}]$, found: $m / z 1100.6420[\mathrm{M}+\mathrm{H}]^{+}$.

IR $v_{\max }(\mathrm{KBr}) \mathrm{cm}^{-1}: 3488,2973,2933,2877,1720$.

${ }^{1} \mathrm{H}-\mathrm{NMR}\left(270 \mathrm{MHz}, \mathrm{CDCl}_{3}\right.$, partial data) $\delta(\mathrm{ppm}): 8.11$ (d, $\left.J=7.0 \mathrm{~Hz}, 2 \mathrm{H}, 4^{\prime \prime}-\mathrm{OCO} P h\right), 7.57$ (m, 1H, 4"-OCOPh), 7.47 7.42 (m, 2H, 4"-OCOPh), 7.34 7.14 (m, 7H, 18-N$\left.P h\left(\mathrm{CH}_{2} P h\right)\right), 6.74 \sim 6.71\left(\mathrm{~m}, 2 \mathrm{H}, 18-\mathrm{N}-\mathrm{Ph}\left(\mathrm{CH}_{2} P h\right)\right), 6.65$ $\left(\mathrm{m}, 1 \mathrm{H}, 18-N-P h\left(\mathrm{CH}_{2} P h\right)\right), 6.01 \sim 5.93(\mathrm{~m}, 2 \mathrm{H}, \mathrm{H}-11, \mathrm{H}-$ 12), $5.65(\mathrm{~m}, 1 \mathrm{H}, \mathrm{H}-10), 5.52(\mathrm{~m}, 1 \mathrm{H}, \mathrm{H}-13), 5.31(\mathrm{~m}, 1 \mathrm{H}$, $\mathrm{H}-15), 5.09$ (d, $\left.J=3.0 \mathrm{~Hz}, 1 \mathrm{H}, \mathrm{H}-1^{\prime \prime}\right), 4.83$ (d, $J=10.3 \mathrm{~Hz}$, $\left.1 \mathrm{H}, \mathrm{H}-4^{\prime \prime}\right), 4.65\left(\mathrm{~m}, 2 \mathrm{H}, 18-N-\left(\mathrm{CH}_{2} P h\right)\right), 4.63(\mathrm{~m}, 1 \mathrm{H}, \mathrm{H}-$ $\left.5^{\prime \prime}\right), 4.25 \sim 4.19\left(\mathrm{~m}, 2 \mathrm{H}, \mathrm{H}-1^{\prime}, \mathrm{H}-1^{\prime \prime \prime}\right), 4.06 \sim 4.02(\mathrm{~m}, 2 \mathrm{H}, \mathrm{H}-$ 5, H-9), 3.74 (m, 1H, H-3), 3.56 (m, 1H, H-2'), 3.51 (s, 3H, 4-OCH$\left.H_{3}\right), 3.32 \sim 3.21$ (m, 2H, H-4', H-5'), 3.09 (d, $J=8.6$ $\mathrm{Hz}, 1 \mathrm{H}, \mathrm{H}-4), 2.53$ (s, 6H, 3'-N(CH$\left.)_{2}\right), 2.39$ (s, 3H, 4"'$\left.\mathrm{NHCH}_{3}\right), 1.30\left(\mathrm{~d}, J=6.5 \mathrm{~Hz}, 3 \mathrm{H}, 15-\mathrm{CH}_{3}\right), 1.19 \sim 1.16(\mathrm{~m}$, $\left.12 \mathrm{H}, 5^{\prime}-\mathrm{CH}_{3}, 3^{\prime \prime}-\mathrm{CH}_{3}, 5^{\prime \prime}-\mathrm{CH}_{3}, 5^{\prime \prime \prime}-\mathrm{CH}_{3}\right), 0.96(\mathrm{~d}, \mathrm{~J}=6.5 \mathrm{~Hz}$, $\left.8-\mathrm{CH}_{3}\right), 0.89$ (m, 1H, H-7).

\section{4"-O-Benzoyl-18- $N$-benzylamino-4"'- $N$-demethyl-18- deoxospiramycin I (3c)}

HR-MS (FAB) (matrix; $m$-NBA): calcd. for $\mathrm{C}_{56} \mathrm{H}_{86} \mathrm{~N}_{3} \mathrm{O}_{14}$ : 1024.6110 [M+H], found: $m / z$ 1024.6108 [M+H] $]^{+}$. 
IR $v_{\max }(\mathrm{KBr}) \mathrm{cm}^{-1}: 3502,2975,2935,2875,1749$, 1722, 1704.

${ }^{1} \mathrm{H}-\mathrm{NMR}\left(270 \mathrm{MHz}, \mathrm{CDCl}_{3}\right.$, partial data) $\delta$ (ppm): 8.10 (d, $J=7.0 \mathrm{~Hz}, 2 \mathrm{H}, 4$ "-OCOPh), $7.57\left(\mathrm{~m}, 1 \mathrm{H}, 4^{\prime \prime}-\mathrm{OCO} P h\right)$, 7.48-7.42 (m, 2H, 4"-OCOPh), 7.35 7.33 (m, 4H, 18- N$\left.\mathrm{CH}_{2} P h\right), 7.27\left(\mathrm{~m}, 1 \mathrm{H}, 18-N-\mathrm{CH}_{2} P h\right), 6.17$ (dd, $J=14.9$, $10.5 \mathrm{~Hz}, 1 \mathrm{H}, \mathrm{H}-11), 5.97$ (dd, $J=14.7,10.8 \mathrm{~Hz}, 1 \mathrm{H}, \mathrm{H}-12$ ), 5.68 (dd, $J=14.9,9.5 \mathrm{~Hz}, 1 \mathrm{H}, \mathrm{H}-10$ ), 5.45 (ddd, $J=15.0$, 11.1, $3.5 \mathrm{~Hz}, 1 \mathrm{H}, \mathrm{H}-13), 5.24$ (m, 1H, H-15), 5.11 (d, $\left.J=3.0 \mathrm{~Hz}, 1 \mathrm{H}, \mathrm{H}-1^{\prime \prime}\right), 4.83$ (d, $\left.J=10.3 \mathrm{~Hz}, 1 \mathrm{H}, \mathrm{H}-4^{\prime \prime}\right), 4.62$ (m, 1H, H-5"), 4.48 4.46 (m, 2H, H-1', H-1"'), 4.15 (dd, $J=10.3,4.3 \mathrm{~Hz}, 1 \mathrm{H}, \mathrm{H}-9$ ), 4.07 (d, $J=8.9 \mathrm{~Hz}, 1 \mathrm{H}, \mathrm{H}-5$ ), $3.83\left(\mathrm{~d}, J=13.2 \mathrm{~Hz}, 1 \mathrm{H}, 18-N-\mathrm{CH}_{2} P h\right), 3.76(\mathrm{~m}, 1 \mathrm{H}, \mathrm{H}-3)$, $3.75\left(\mathrm{~d}, J=13.2 \mathrm{~Hz}, 1 \mathrm{H}, 18-\mathrm{N}-\mathrm{CH}_{2} P h\right), 3.56(\mathrm{dd}, J=10.3$, $\left.7.0 \mathrm{~Hz}, 1 \mathrm{H}, \mathrm{H}-2^{\prime}\right), 3.50$ (s, 3H, 4-OCH $\left.H_{3}\right), 3.32(\mathrm{~m}, 2 \mathrm{H}, \mathrm{H}-$ 4', H-5'), 3.18 (m, 1H, H-5'"), 3.08 (d, J=8.4 Hz, 1H, H-4), $2.53\left(\mathrm{~s}, 6 \mathrm{H}, 3^{\prime}-\mathrm{N}\left(\mathrm{CH}_{3}\right)_{2}\right), 2.40$ (s, 3H, 4"'- $\left.\mathrm{NHCH}_{3}\right), 1.29$ (d, $\left.J=6.5 \mathrm{~Hz}, 3 \mathrm{H}, 15-\mathrm{CH}_{3}\right), 1.25-1.17$ (m, 12H, 5'-CH 3 , 3"$\left.\mathrm{CH}_{3}, 5^{\prime \prime}-\mathrm{CH}_{3}, 5^{\prime \prime \prime}-\mathrm{CH}_{3}\right), 0.97$ (d, J=6.5 Hz, 8- $\left.\mathrm{CH}_{3}\right), 0.91$ (m, $1 \mathrm{H}, \mathrm{H}-7)$.

\section{4"-O-Benzoyl-4"'- $N$-demethyl-18-( $N, N$-di-2- picolyl)amino-18-deoxospiramycin I (3d)}

HR-MS (FAB) (matrix; $m$-NBA): calcd. for $\mathrm{C}_{61} \mathrm{H}_{90} \mathrm{~N}_{5} \mathrm{O}_{14}$ : $1116.6484[\mathrm{M}+\mathrm{H}]$, found: $m / z 1024.6448[\mathrm{M}+\mathrm{H}]^{+}$.

IR $v_{\max }(\mathrm{KBr}) \mathrm{cm}^{-1}: 3457,2935,1722$.

${ }^{1} \mathrm{H}-\mathrm{NMR}\left(270 \mathrm{MHz}, \mathrm{CDCl}_{3}\right.$, partial data) $\delta(\mathrm{ppm})$ : $8.62 \sim 8.54\left(\mathrm{~m}, 2 \mathrm{H}, 18-\mathrm{N}-\mathrm{CH}_{2}\right.$ (2-pyridine)), 8.12 8.08 (m, $2 \mathrm{H}, \quad 4 "-\mathrm{OCOPh}), \quad 7.74 \sim 7.65 \quad\left(\mathrm{~m}, \quad 2 \mathrm{H}, \quad 18-\mathrm{N}-\mathrm{CH}_{2}(2-\right.$ pyridine)), $7.61 \sim 7.55\left(\mathrm{~m}, 3 \mathrm{H}, 18-\mathrm{N}-\mathrm{CH}_{2}\right.$ (2-pyridine), 4"OCOPh $), 7.48 \sim 7.42\left(\mathrm{~m}, 2 \mathrm{H}, 44^{\prime \prime}-\mathrm{OCOPh}\right), 7.23 \sim 7.12(\mathrm{~m}$, $2 \mathrm{H}, 18-\mathrm{N}-\mathrm{CH}_{2}(2-$ pyridine $\left.)\right), 5.94(\mathrm{~m}, 1 \mathrm{H}, \mathrm{H}-11), 5.81(\mathrm{~m}$, $1 \mathrm{H}, \mathrm{H}-12), 5.62$ (dd, $J=14.0,9.7 \mathrm{~Hz}, 1 \mathrm{H}, \mathrm{H}-10), 5.42$ (m, $1 \mathrm{H}, \mathrm{H}-13), 5.25$ (m, 1H, H-15), 5.05 (d, J=3.0 Hz, 1H, H$\left.1^{\prime \prime}\right), 4.81$ (d, $\left.J=9.7 \mathrm{~Hz}, 1 \mathrm{H}, \mathrm{H}-4^{\prime \prime}\right), 4.60$ (m, 1H, H-5"), 4.30 (dd, $J=7.8 \mathrm{~Hz}, 1 \mathrm{H}, \mathrm{H}-1^{\prime}$ ), 4.16 (m, 1H, H-1"'), 4.00 3.88 (m, 5H, H-3, H-5, H-9, 18- $\mathrm{N}-\mathrm{CH}_{2}$ (2-pyridine)), 3.59 (m, $\left.1 \mathrm{H}, \mathrm{H}-2^{\prime}\right), 3.52$ (s, 3H, 4-OCH $\left.{ }_{3}\right), 3.43$ (d, $J=14.0 \mathrm{~Hz}, 2 \mathrm{H}$, $18-\mathrm{N}-\mathrm{CH}_{2}(2-$ pyridine $)$ ), $3.27 \sim 3.11\left(\mathrm{~m}, 2 \mathrm{H}, \mathrm{H}-4^{\prime}, \mathrm{H}-5^{\prime}\right)$, $2.51\left(\mathrm{~s}, 6 \mathrm{H}, 3^{\prime}-\mathrm{N}\left(\mathrm{CH}_{3}\right)_{2}\right), 2.41$ (s, 3H, 4'"-- $\left.\mathrm{NHCH}_{3}\right), 1.31$ (d, $\left.J=6.5 \mathrm{~Hz}, 3 \mathrm{H}, 15-\mathrm{CH}_{3}\right), 1.24 \sim 1.15\left(\mathrm{~m}, 9 \mathrm{H}, 5^{\prime}-\mathrm{CH}_{3}, 3^{\prime \prime}-\right.$ $\mathrm{CH}_{3}, 5^{\prime \prime}-\mathrm{CH}_{3}$ ), 1.09 (d, $\left.J=6.2 \mathrm{~Hz}, 3 \mathrm{H}, 5^{\prime \prime \prime}-\mathrm{CH}_{3}\right), 0.93$ (d, $\left.J=6.8 \mathrm{~Hz}, 3 \mathrm{H}, 8-\mathrm{CH}_{3}\right), 0.84(\mathrm{~m}, 1 \mathrm{H}, \mathrm{H}-7)$.

\section{4"-O-Benzoyl-4"'- $N$-demethyl-18-( $N, N$-di-3- picolyl)amino-18-deoxospiramycin I (3e)}

HR-MS (FAB) (matrix; $m$-NBA): calcd. for $\mathrm{C}_{61} \mathrm{H}_{90} \mathrm{~N}_{5} \mathrm{O}_{14}$ : $1116.6484[\mathrm{M}+\mathrm{H}]$, found: $m / z 1024.6454[\mathrm{M}+\mathrm{H}]^{+}$.

IR $v_{\max }(\mathrm{KBr}) \mathrm{cm}^{-1}: 34902973,2935,2875,1722$.

${ }^{1} \mathrm{H}-\mathrm{NMR}\left(270 \mathrm{MHz}, \mathrm{CDCl}_{3}\right.$, partial data) $\delta(\mathrm{ppm})$ : $8.57 \sim 8.56\left(\mathrm{~m}, 2 \mathrm{H}, 18-\mathrm{N}-\mathrm{CH}_{2}\right.$ (3-pyridine)), 8.55 8.49 (m,
$2 \mathrm{H}, \quad 18-\mathrm{N}-\mathrm{CH}_{2}(3$-pyridine $\left.)\right), \quad 8.12 \sim 8.08 \quad(\mathrm{~m}, \quad 2 \mathrm{H}, \quad 4 "-$ $\mathrm{OCOPh}), 7.79 \sim 7.76\left(\mathrm{~m}, 2 \mathrm{H}, 18-\mathrm{N}-\mathrm{CH}_{2}(3\right.$-pyridine $\left.)\right), 7.57$ (m, 1H, 4"-OCOPh), 7.45 (m, 2H, 4"-OCOPh), 7.32 7.29 (m, 2H, 18- $\mathrm{N}-\mathrm{CH}_{2}$ (3-pyridine)), 6.03 5.96 (m, 2H, H-11, $\mathrm{H}-12), 5.64$ (m, 1H, H-10), 5.55 (m, 1H, H-13), 5.31 (m, $1 \mathrm{H}, \mathrm{H}-15), 5.07$ (d, $\left.J=3.0 \mathrm{~Hz}, 1 \mathrm{H}, \mathrm{H}-1^{\prime \prime}\right), 4.82$ (d, $J=10.3$ Hz, 1H, H-4"), 4.61 (m 1H, H-5"), 4.34 4.27 (m, 2H, H1', H-1"'), $4.03 \sim 3.92$ (m, 2H, H-5, H-9), 3.73 (m, 1H, H3), $3.71\left(\mathrm{~d}, J=13.0 \mathrm{~Hz}, 2 \mathrm{H}, 18-\mathrm{N}-\mathrm{CH}_{2}(3-\right.$ pyridine $\left.)\right), 3.55$ (m, 1H, H-2'), 3.49 (m, 3H, 4-OCH $), 3.73$ (d, $J=13.0 \mathrm{~Hz}$, $2 \mathrm{H}, 18-\mathrm{N}-\mathrm{CH}_{2}$ (3-pyridine)), 3.15 3.05 (m, 2H, H-4', H$\left.5^{\prime}\right), 2.52\left(\mathrm{~s}, 6 \mathrm{H}, 3^{\prime}-\mathrm{N}\left(\mathrm{CH}_{3}\right)_{2}\right), 2.40\left(\mathrm{~s}, 3 \mathrm{H}, 4^{\prime \prime \prime}-\mathrm{NHCH}_{3}\right)$, $1.31\left(\mathrm{~d}, J=6.5 \mathrm{~Hz}, 3 \mathrm{H}, 15-\mathrm{CH}_{3}\right), 1.18 \sim 1.14\left(\mathrm{~m}, 12 \mathrm{H}, 5^{\prime}-\right.$ $\left.\mathrm{CH}_{3}, 3^{\prime \prime}-\mathrm{CH}_{3}, 5^{\prime \prime}-\mathrm{CH}_{3}, 5^{\prime \prime \prime}-\mathrm{CH}_{3}\right), 0.95(\mathrm{~d}, J=6.5 \mathrm{~Hz}, 3 \mathrm{H}, 8-$ $\left.\mathrm{CH}_{3}\right), 0.84(\mathrm{~m}, 1 \mathrm{H}, \mathrm{H}-7)$.

\section{4"-O-Benzoyl-4"'- $\mathrm{N}$-demethyl-18-(4-phenylpiperazin-1- yl)-18-deoxospiramycin I (3f)}

HR-MS (FAB) (matrix; $m$-NBA): calcd. for $\mathrm{C}_{59} \mathrm{H}_{91} \mathrm{~N}_{4} \mathrm{O}_{14}$ : $1079.6532[\mathrm{M}+\mathrm{H}]$, found: $m / z 1079.6543[\mathrm{M}+\mathrm{H}]^{+}$.

IR $v_{\max }(\mathrm{KBr}) \mathrm{cm}^{-1}: 3494,2973,2935,2875,1722$.

${ }^{1} \mathrm{H}-\mathrm{NMR}\left(270 \mathrm{MHz}, \mathrm{CDCl}_{3}\right.$, partial data) $\delta(\mathrm{ppm}): 8.10$ $\left(\mathrm{d}, J=7.0 \mathrm{~Hz}, 2 \mathrm{H}, 4^{\prime \prime}-\mathrm{OCO} P h\right), 7.57\left(\mathrm{~m}, 1 \mathrm{H}, 4^{\prime \prime}-\mathrm{OCO} P h\right)$, $7.48 \sim 7.42\left(\mathrm{~m}, 2 \mathrm{H}, 4^{\prime \prime}-\mathrm{OCOPh}\right), 7.29 \sim 7.23$ (m, 2H, 18-N$P h), 6.94 \sim 6.92(\mathrm{~m}, 2 \mathrm{H}, 18-N-P h), 6.85(\mathrm{~m}, 1 \mathrm{H}, 18-N-P h)$, 6.20 (dd, $J=14.8,10.5 \mathrm{~Hz}, 1 \mathrm{H}, \mathrm{H}-11), 6.01$ (dd, $J=14.8$, $10.5 \mathrm{~Hz}, 1 \mathrm{H}, \mathrm{H}-12$ ), 5.70 (dd, $J=14.9,9.5 \mathrm{~Hz}, 1 \mathrm{H}, \mathrm{H}-10$ ), 5.51 (ddd, $J=15.0,11.1,3.5 \mathrm{~Hz}, 1 \mathrm{H}, \mathrm{H}-13), 5.18(\mathrm{~m}, 1 \mathrm{H}$, H-15), 5.14 (d, $\left.J=3.0 \mathrm{~Hz}, 1 \mathrm{H}, \mathrm{H}-1^{\prime \prime}\right), 4.83$ (d, $J=10.3 \mathrm{~Hz}$, $\left.1 \mathrm{H}, \mathrm{H}-4^{\prime \prime}\right), 4.63$ (m, 1H, H-5"), 4.45 4.48 (m, 2H, H-1', H$\left.1^{\prime \prime \prime}\right), 4.17 \sim 4.08$ (m, 2H, H-5), 3.78 (d, $J=10.5 \mathrm{~Hz}, 1 \mathrm{H}, \mathrm{H}-$ 3), $3.59\left(\mathrm{dd}, J=10.3,7.0 \mathrm{~Hz}, 1 \mathrm{H}, \mathrm{H}-2^{\prime}\right), 3.51$ (s, $3 \mathrm{H}, 4-$ $\left.\mathrm{OCH}_{3}\right), 2.54\left(\mathrm{~s}, 6 \mathrm{H}, 3^{\prime}-\mathrm{N}\left(\mathrm{CH}_{3}\right)_{2}\right), 2.41\left(\mathrm{~s}, 3 \mathrm{H}, 4^{\prime \prime \prime}-\right.$ $\left.\mathrm{NH}\left(\mathrm{CH}_{3}\right)\right), 1.31 \sim 1.25\left(\mathrm{~m}, 12 \mathrm{H}, 15-\mathrm{CH}_{3}, 5^{\prime}-\mathrm{CH}_{3}, 3^{\prime \prime}-\mathrm{CH}_{3}\right.$, $\left.5^{\prime \prime}-\mathrm{CH}_{3}\right), 1.18$ (d, $\left.J=6.5 \mathrm{~Hz}, 3 \mathrm{H}, 5^{\prime \prime \prime}-\mathrm{CH}_{3}\right), 0.98$ (d, $J=6.5$ $\left.\mathrm{Hz}, 8-\mathrm{CH}_{3}\right), 0.93$ (m, 1H, H-7).

\section{4"-O-Benzoyl-4"'-N-demethyl-18-(indolin-1-yl)-18- deoxospiramycin I (3g)}

HR-MS (FAB) (matrix; $m$-NBA): calcd. for $\mathrm{C}_{57} \mathrm{H}_{86} \mathrm{~N}_{3} \mathrm{O}_{14}$ : $1036.6110[\mathrm{M}+\mathrm{H}]$, found: $m / z 1036.6145[\mathrm{M}+\mathrm{H}]^{+}$.

IR $v_{\max }(\mathrm{KBr}) \mathrm{cm}^{-1}: 3490,2935,1733$.

${ }^{1} \mathrm{H}-\mathrm{NMR}\left(270 \mathrm{MHz}, \mathrm{CDCl}_{3}\right.$, partial data) $\delta$ (ppm): 8.07 (d, $\left.J=7.2 \mathrm{~Hz}, 2 \mathrm{H}, 4^{\prime \prime}-\mathrm{OCO} P h\right), 7.53\left(\mathrm{~m}, 1 \mathrm{H}, 4^{\prime \prime}-\mathrm{OCO} P h\right)$, $7.43 \sim 7.38$ (m, 2H, 4"-OCOPh), 7.03 (d, $J=7.0 \mathrm{~Hz}, 1 \mathrm{H}, 18-$ $N$-indoline), 6.99 (d, $J=8.0 \mathrm{~Hz}, 1 \mathrm{H}, 18-N$-indoline), 6.58 (t, $J=7.3 \mathrm{~Hz}, 1 \mathrm{H}, 18-N$-indoline), 6.49 (d, $J=7.8 \mathrm{~Hz}, 1 \mathrm{H}, 18-$ $N$-indoline), $6.00 \sim 5.93$ (m, 2H, H-11, H-12), 5.63 (dd, $J=13.8,9.5 \mathrm{~Hz}, 1 \mathrm{H}, \mathrm{H}-10$ ), 5.47 (ddd, $J=13.9,11.1,4.9$ $\mathrm{Hz}, 1 \mathrm{H}, \mathrm{H}-13), 5.24$ (m, 1H, H-15), 5.09 (d, $J=4.1 \mathrm{~Hz}, 1 \mathrm{H}$, H-1"), 4.80 (d, $\left.J=10.3 \mathrm{~Hz}, 1 \mathrm{H}, \mathrm{H}-4^{\prime \prime}\right), 4.63$ (m, 1H, H-5"), 
$4.55\left(\mathrm{~d}, J=7.6 \mathrm{~Hz}, 1 \mathrm{H}, \mathrm{H}-1^{\prime}\right), 4.19$ (m, 1H, H-5), 3.96 (d, $\left.J=8.6 \mathrm{~Hz}, 1 \mathrm{H}, \mathrm{H}-1^{\prime \prime \prime}\right), 3.90$ (dd, $J=9.5,3.8 \mathrm{~Hz}, 1 \mathrm{H}, \mathrm{H}-9$ ), 3.75 (d, $J=10.5 \mathrm{~Hz}, 1 \mathrm{H}, \mathrm{H}-3$ ), 3.57 (dd, $J=10.3,7.6 \mathrm{~Hz}$, $\left.1 \mathrm{H}, \mathrm{H}-2^{\prime}\right), 3.50\left(\mathrm{~s}, 3 \mathrm{H}, 4-\mathrm{OCH}_{3}\right), 2.52\left(\mathrm{~s}, 6 \mathrm{H}, 3^{\prime}-\mathrm{N}\left(\mathrm{CH}_{3}\right)_{2}\right)$, 2.35 (s, 3H, 4"'-- $\left.\mathrm{NHCH}_{3}\right), 1.25$ (d, $\left.J=6.5 \mathrm{~Hz}, 3 \mathrm{H}, 15-\mathrm{CH}_{3}\right)$, $1.16 \sim 1.12\left(\mathrm{~m}, 9 \mathrm{H}, 5^{\prime}-\mathrm{CH}_{3}, 3^{\prime \prime}-\mathrm{CH}_{3}, 5^{\prime \prime \prime}-\mathrm{CH}_{3}\right), 1.09(\mathrm{~d}$, $\left.J=6.2 \mathrm{~Hz}, 3 \mathrm{H}, 5^{\prime \prime}-\mathrm{CH}_{3}\right), 0.94\left(\mathrm{~d}, J=6.5 \mathrm{~Hz}, 3 \mathrm{H}, 8-\mathrm{CH}_{3}\right)$, $0.86(\mathrm{~m}, 1 \mathrm{H}, \mathrm{H}-7)$.

\section{4"-O-Benzoyl-4"'-N-demethyl-18-(isoindolin-2-yl)-18- deoxospiramycin I (3h)}

HR-MS (FAB) (matrix; $m$-NBA): calcd. for $\mathrm{C}_{57} \mathrm{H}_{85} \mathrm{~N}_{3} \mathrm{O}_{14} \mathrm{Na}$ : 1058.5929, found: $\mathrm{m} / \mathrm{z} 1058.5973$ $[\mathrm{M}+\mathrm{Na}]^{+}$.

IR $v_{\max }(\mathrm{KBr}) \mathrm{cm}^{-1}: 3469,2967,2935,1720$.

${ }^{1} \mathrm{H}-\mathrm{NMR}\left(270 \mathrm{MHz}, \mathrm{CDCl}_{3}\right.$, partial data) $\delta$ (ppm): 8.09 (d, $\left.J=7.0 \mathrm{~Hz}, 2 \mathrm{H}, 4^{\prime \prime}-\mathrm{OCOPh}\right), 7.57$ (m, 1H, 4"-OCOPh), $7.54 \sim 7.48\left(\mathrm{~m}, 2 \mathrm{H}, 4^{\prime \prime}-\mathrm{OCOPh}\right), 7.20(\mathrm{~s}, 4 \mathrm{H}, 18-\mathrm{N}-$ isoindoline), 6.18 (dd, $J=15.0,10.3 \mathrm{~Hz}, 1 \mathrm{H}, \mathrm{H}-11), 6.00$ (dd, $J=14.610 .5 \mathrm{~Hz}, 1 \mathrm{H}, \mathrm{H}-12), 5.70$ (dd, $J=14.9,9.7 \mathrm{~Hz}$, $1 \mathrm{H}, \mathrm{H}-10$ ), 5.56 (ddd, $J=14.7,10.7,4.1 \mathrm{~Hz}, 1 \mathrm{H}, \mathrm{H}-13$ ), $5.22(\mathrm{~m}, 1 \mathrm{H}, \mathrm{H}-15), 5.12$ (d, J=3.2 Hz, 1H, H-1"), 4.83 (d, $\left.J=10.3 \mathrm{~Hz}, 1 \mathrm{H}, \mathrm{H}-4^{\prime \prime}\right), 4.69$ (m, 2H, H-5"), 4.63 (d, $J=7.6$ $\left.\mathrm{Hz}, 1 \mathrm{H}, \mathrm{H}-1^{\prime}\right), 4.52$ (m, 1H, H-1"'), $4.21 \sim 4.18$ (m, 2H, H9, H-5), 3.98 (s, 4H, 18- $N$-isoindoline), 3.77 (d, $J=10.5 \mathrm{~Hz}$, $1 \mathrm{H}, \mathrm{H}-3), 3.60$ (dd, $\left.J=10.5,7.6 \mathrm{~Hz}, 1 \mathrm{H}, \mathrm{H}-2^{\prime}\right), 3.63$ (s, $3 \mathrm{H}$, 4-OCH $H_{3}$ ), 3.43 3.35 (m, 2H, H-4', H-5'), 2.60 (s, 6H, 3'$\left.\mathrm{N}\left(\mathrm{CH}_{3}\right)_{2}\right), 2.46\left(\mathrm{~s}, 3 \mathrm{H}, 4^{\prime \prime \prime}-\mathrm{NHCH}_{3}\right), 1.28(\mathrm{~d}, J=6.5 \mathrm{~Hz}, 3 \mathrm{H}$, $\left.15-\mathrm{CH}_{3}\right), 1.28 \sim 1.22\left(\mathrm{~m}, 12 \mathrm{H}, 5^{\prime}-\mathrm{CH}_{3}, 3^{\prime \prime}-\mathrm{CH}_{3}, 5^{\prime \prime}-\mathrm{CH}_{3}, 5^{\prime \prime \prime}-\right.$ $\left.\mathrm{CH}_{3}\right), 1.00\left(\mathrm{~d}, J=6.5 \mathrm{~Hz}, 3 \mathrm{H}, 8-\mathrm{CH}_{3}\right), 0.96(\mathrm{~m}, 1 \mathrm{H}, \mathrm{H}-7)$.

\section{4"-O-Benzoyl-4"'- $N$-demethyl-18-(1,2,3,4-}

\section{tetrahydroquinolin-1-yl)-18-deoxospiramycin I (3i)}

HR-MS (FAB) (matrix; $m$-NBA): calcd. for $\mathrm{C}_{58} \mathrm{H}_{88} \mathrm{~N}_{3} \mathrm{O}_{14}$ : $1050.6266[\mathrm{M}+\mathrm{H}]$, found: $m / z 1050.6211[\mathrm{M}+\mathrm{H}]^{+}$.

IR $v_{\max }(\mathrm{KBr}) \mathrm{cm}^{-1}:$ 3491, 2967, 2935, 2865, 1720.

${ }^{1} \mathrm{H}-\mathrm{NMR}\left(270 \mathrm{MHz}, \mathrm{CDCl}_{3}\right.$, partial data) $\delta$ (ppm): 8.10 (d, $\left.J=7.0 \mathrm{~Hz}, 2 \mathrm{H}, 4^{\prime \prime}-\mathrm{OCO} P h\right), 7.58\left(\mathrm{~m}, 1 \mathrm{H}, 4^{\prime \prime}-\mathrm{OCO} P h\right)$, $7.48 \sim 7.42$ (m, 2H, 4"-OCOPh), 7.02 (t, $J=7.3 \mathrm{~Hz}, 1 \mathrm{H}, 18-$ $N$-1,2,3,4-tetrahydroquinoline), $6.96(\mathrm{~d}, J=7.3 \mathrm{~Hz}, 1 \mathrm{H}, 18-$ $N$-1,2,3,4-tetrahydroquinoline), $6.62(\mathrm{~d}, J=8.1 \mathrm{~Hz}, 1 \mathrm{H}, 18-$ $N$-1,2,3,4-tetrahydroquinoline), $6.55(\mathrm{t}, J=7.3 \mathrm{~Hz}, 1 \mathrm{H}, 18$ $N$-1,2,3,4-tetrahydroquinoline), 6.11 65.98 (m, 2H, H-11, H-12), 5.68 (dd, $J=14.2,9.7 \mathrm{~Hz}, 1 \mathrm{H}, \mathrm{H}-10$ ), 5.50 (ddd, $J=14.4,11.0,3.2 \mathrm{~Hz}, 1 \mathrm{H}, \mathrm{H}-13), 5.30$ (m, 1H, H-15), 5.14 (d, $\left.J=3.0 \mathrm{~Hz}, 1 \mathrm{H}, \mathrm{H}-\mathrm{1}^{\prime \prime}\right), 4.84$ (d, $\left.J=10.0 \mathrm{~Hz}, 1 \mathrm{H}, \mathrm{H}-4^{\prime \prime}\right)$, $4.64\left(\mathrm{~m}, 1 \mathrm{H}, \mathrm{H}-5^{\prime \prime}\right), 4.57$ (d, $\left.J=7.6 \mathrm{~Hz} 1 \mathrm{H}, \mathrm{H}-1^{\prime}\right), 4.25$ (d, $J=8.9 \mathrm{~Hz}, 1 \mathrm{H}, \mathrm{H}-5), 3.90$ (d, $\left.J=7.8 \mathrm{~Hz}, 1 \mathrm{H}, \mathrm{H}-1^{\prime \prime \prime}\right), 3.80 \sim$ 3.73 (m, 2H, H-3, H-9), 3.58 (dd, $J=10.3,7.6 \mathrm{~Hz}, 1 \mathrm{H}, \mathrm{H}-$ $\left.2^{\prime}\right), 3.53\left(\mathrm{~s}, 3 \mathrm{H}, 4-\mathrm{OCH}_{3}\right), 3.48 \sim 3.28(\mathrm{~m}, 4 \mathrm{H}, 18-\mathrm{N}-$ 1,2,3,4-tetrahydroquinoline, H-4', H-5'), 3.09 (d, $J=8.4 \mathrm{~Hz}$,
1H, H-4), 2.55 (s, 6H, 3'-N( $\left.\left.\mathrm{CH}_{3}\right)_{2}\right), 2.41\left(\mathrm{~s}, 3 \mathrm{H}, 4^{\prime \prime \prime}-\right.$ $\left.\mathrm{NHCH}_{3}\right), 1.30\left(\mathrm{~d}, J=6.5 \mathrm{~Hz}, 3 \mathrm{H}, 15-\mathrm{CH}_{3}\right), 1.20-1.14(\mathrm{~m}$, $\left.12 \mathrm{H}, 5^{\prime}-\mathrm{CH}_{3}, 3^{\prime \prime}-\mathrm{CH}_{3}, 5^{\prime \prime}-\mathrm{CH}_{3}, 5^{\prime \prime \prime}-\mathrm{CH}_{3}\right), 0.94$ (d, $J=6.5 \mathrm{~Hz}$, $\left.3 \mathrm{H}, 8-\mathrm{CH}_{3}\right), 0.84$ (m, 1H, H-7).

\section{4"-O-Benzoyl-4"'-N-demethyl-18-(1,2,3,4-}

tetrahydroisoquinolin-2-yl)-18-deoxospiramycin I (3j) HR-MS (FAB) (matrix; $m$-NBA): calcd. for $\mathrm{C}_{58} \mathrm{H}_{88} \mathrm{~N}_{3} \mathrm{O}_{14}$ : $1050.6266[\mathrm{M}+\mathrm{H}]$, found: $m / z$ 1050. $6257[\mathrm{M}+\mathrm{H}]^{+}$.

IR $v_{\max }(\mathrm{KBr}) \mathrm{cm}^{-1}: 3488,2937,1720$.

${ }^{1} \mathrm{H}-\mathrm{NMR}\left(270 \mathrm{MHz}, \mathrm{CDCl}_{3}\right) \delta(\mathrm{ppm}): 8.09(\mathrm{~d}, J=7.0 \mathrm{~Hz}$, $\left.2 \mathrm{H}, 4^{\prime \prime}-\mathrm{OCO} P h\right), 7.56\left(\mathrm{~m}, 1 \mathrm{H}, 4^{\prime \prime}-\mathrm{OCO} P\right.$ ), 7.44 (m, 2H, 4"-OCOPh $), \quad 7.07 \quad(\mathrm{~m}, \quad 4 \mathrm{H}, \quad 18-\mathrm{N}$-1,2,3,4-tetrahydroisoquinoline), 6.07 5.95 (m, 2H, H-11, H-12), 5.92 (dd, $J=14.2,10.5 \mathrm{~Hz}, 1 \mathrm{H}, \mathrm{H}-10), 5.65$ (dd, $J=14.4,9.7 \mathrm{~Hz}, 1 \mathrm{H}$, H-13), 5.13 (m, 1H, H-15), 5.12 (d, $J=3.0 \mathrm{~Hz}, 1 \mathrm{H}, \mathrm{H}-1^{\prime \prime}$ ), 4.82 (d, $\left.J=10.3 \mathrm{~Hz}, 1 \mathrm{H}, \mathrm{H}-4^{\prime \prime}\right), 4.53$ (m, 1H, H-5"), 4.56 (d, $\left.J=7.6 \mathrm{~Hz}, 1 \mathrm{H}, \mathrm{H}-1^{\prime}\right), 4.39$ (dd, $J=9.3,2.2 \mathrm{~Hz}, 1 \mathrm{H}, \mathrm{H}-1^{\prime \prime \prime}$ ), $4.16 \sim 4.13$ (m, 2H, H-5, H-9), 4.07 (d, J=10.8 Hz, 1H, H3), 3.50 (dd, $\left.J=10.5,7.6 \mathrm{~Hz}, 1 \mathrm{H}, \mathrm{H}-2^{\prime}\right), 3.45$ (s, 3H, 4$\mathrm{OCH}_{3}$ ), 3.34 3.11 (m, 2H, H-4', H-5'), 2.53 (s, 6H, 3'$\left.\mathrm{N}\left(\mathrm{CH}_{3}\right)_{2}\right), 2.40$ (s, 3H, 4"'-NHCH$\left.H_{3}\right), 1.29$ (d, $J=6.5 \mathrm{~Hz}, 3 \mathrm{H}$, $\left.15-\mathrm{CH}_{3}\right), 1.22 \sim 1.16\left(\mathrm{~m}, 12 \mathrm{H}, 5^{\prime}-\mathrm{CH}_{3}, 3^{\prime \prime}-\mathrm{CH}_{3}, 5^{\prime \prime}-\mathrm{CH}_{3}, 5^{\prime \prime \prime}-\right.$ $\left.\mathrm{CH}_{3}\right), 0.95\left(\mathrm{~d}, J=6.2 \mathrm{~Hz}, 3 \mathrm{H}, 8-\mathrm{CH}_{3}\right), 0.87$ (m, 1H, H-7).

\section{4"'-N-Demethyl-18-(indolin-1-yl)-18-deoxospiramycin I}

(8)

Title compound 8 was prepared from compound $\mathbf{4}$ by Fmoc protection, $N$-amination, Fmoc deprotection, and methanolysis (37\%, 4 steps).

HR-MS (FAB) (matrix; $m$-NBA): calcd. for $\mathrm{C}_{58} \mathrm{H}_{88} \mathrm{~N}_{3} \mathrm{O}_{14}$ : $932.5848[\mathrm{M}+\mathrm{H}]$, found: $m / z 932.5814$ $[\mathrm{M}+\mathrm{H}]^{+}$.

IR $v_{\max }(\mathrm{KBr}) \mathrm{cm}^{-1}: 3478,2933,1727$.

${ }^{1} \mathrm{H}-\mathrm{NMR}\left(270 \mathrm{MHz}, \mathrm{CDCl}_{3}\right.$, partial data) $\delta$ (ppm): 7.06 (t, $J=7.5 \mathrm{~Hz}, 1 \mathrm{H}, 18-N$-indoline), 7.03 (d, $J=7.5 \mathrm{~Hz}, 1 \mathrm{H}$, 18 - $N$-indoline), 6.62 (t, $J=7.5 \mathrm{~Hz}, 1 \mathrm{H}, 18-N$-indoline), 6.50 (d, $J=7.5 \mathrm{~Hz}, 1 \mathrm{H}, 18-N$-indoline), $6.02 \sim 5.97$ (m, $2 \mathrm{H}, \mathrm{H}-$ 11, H-12), 5.65 (dd, $J=14.0,10.0 \mathrm{~Hz}, 1 \mathrm{H}, \mathrm{H}-10), 5.47$ (m, $1 \mathrm{H}, \mathrm{H}-13), 5.28$ (m, 1H, H-15), 5.06 (d, $J=3.0 \mathrm{~Hz}, 1 \mathrm{H}, \mathrm{H}-$ $\left.1^{\prime \prime}\right), 4.54\left(\mathrm{~d}, J=7.3 \mathrm{~Hz}, 1 \mathrm{H}, \mathrm{H}-1^{\prime}\right), 4.22(\mathrm{~d}, J=8.9 \mathrm{~Hz}, 1 \mathrm{H}$, H-5"), 3.23 (m, 1H, H-1"'), 3.90 (dd, $J=9.5,3.8 \mathrm{~Hz}, 1 \mathrm{H}, \mathrm{H}-$ 9), 3.79 (d, $J=10.5 \mathrm{~Hz}, 1 \mathrm{H}, \mathrm{H}-3), 3.57$ (m, 1H, H-2'), 3.51 (s, 3H, 4-OCH $\mathrm{OCH}_{3}, 2.47$ (s, 6H, 3'-N( $\left.\left.\mathrm{CH}_{3}\right)_{2}\right), 2.40$ (s, 3H, 4'"$\left.\mathrm{NHCH}_{3}\right), 1.30 \sim 1.22\left(\mathrm{~m}, 12 \mathrm{H}, 15-\mathrm{CH}_{3}, 5^{\prime}-\mathrm{CH}_{3}, 5^{\prime \prime \prime}-\mathrm{CH}_{3}\right)$, 1.14 (d, $\left.J=6.5 \mathrm{~Hz}, 3 \mathrm{H}, 5^{\prime \prime}-\mathrm{CH}_{3}\right), 0.96(\mathrm{~d}, J=6.5 \mathrm{~Hz}, 3 \mathrm{H}, 8-$ $\left.\mathrm{CH}_{3}\right), 0.84(\mathrm{~m}, 1 \mathrm{H}, \mathrm{H}-7)$.

4"-O-Benzoyl-18-(indolin-1-yl)-18-deoxospiramycin I (9) Title compound 9 was prepared from compound 5 by $\mathrm{N}$ amination followed by methanolysis (84\%, 2 steps). 
HR-MS (FAB) (matrix; $m$-NBA): calcd. for $\mathrm{C}_{58} \mathrm{H}_{88} \mathrm{~N}_{3} \mathrm{O}_{14}$ : $1050.6266[\mathrm{M}+\mathrm{H}]$, found: $m / z 1050.6223$ $[\mathrm{M}+\mathrm{H}]^{+}$.

IR $v_{\max }(\mathrm{KBr}) \mathrm{cm}^{-1}: 3450,2935,1724$.

${ }^{1} \mathrm{H}-\mathrm{NMR}\left(270 \mathrm{MHz}, \mathrm{CDCl}_{3}\right.$, partial data) $\delta(\mathrm{ppm}): 8.09$ (d, J=7.2 Hz, 2H, 4"-OCOPh), 7.57 (m, 1H, 4"-OCOPh), $7.47 \sim 7.42\left(\mathrm{~m}, 2 \mathrm{H}, 4^{\prime \prime}-\mathrm{OCO} P h\right), 7.05$ (t, J=7.5 Hz, 1H, 18$N$-indoline), 7.03 (d, $J=8.0 \mathrm{~Hz}, 1 \mathrm{H}, 18-N$-indoline), 6.61 (t, $J=7.3 \mathrm{~Hz}, 1 \mathrm{H}, 18-N$-indoline), 6.52 (d, $J=7.8 \mathrm{~Hz}, 1 \mathrm{H}, 18-$ $N$-indoline), $6.03 \sim 5.96$ (m, 2H, H-11, H-12), 5.67 (dd, $J=14.0,10.0 \mathrm{~Hz}, 1 \mathrm{H}, \mathrm{H}-10), 5.47$ (ddd, $J=13.9,11.1,4.9$ $\mathrm{Hz}, 1 \mathrm{H}, \mathrm{H}-13), 5.28$ (m, 1H, H-15), 5.13 (d, J=3.5 Hz, 1H, H-1"), 4.83 (d, J=10.3 Hz, 1H, H-4"), 4.63 (m, 1H, H-5"), 4.57 (d, $J=7.3 \mathrm{~Hz}, 1 \mathrm{H}, \mathrm{H}-1^{\prime}$ ), 4.46 (br s, 2H, 18-Nindoline), 3.23 (d, $J=8.6 \mathrm{~Hz}, 1 \mathrm{H}, \mathrm{H}-1^{\prime \prime \prime}$ ), 4.03 (br s, 2H, 18$N$-indoline), 3.86 (m, 1H, H-9), 4.19 (d, $J=8.1 \mathrm{~Hz}, 1 \mathrm{H}, \mathrm{H}-$ 5), 3.79 (d, J=10.5 Hz, 1H, H-3), 3.59 (m, 1H, H-2'), 3.53 $\left(\mathrm{s}, 3 \mathrm{H}, 4-\mathrm{OCH}_{3}\right), 2.55\left(\mathrm{~s}, 6 \mathrm{H}, 3^{\prime}-\mathrm{N}\left(\mathrm{CH}_{3}\right)_{2}\right), 2.25\left(\mathrm{~s}, 6 \mathrm{H}, 4^{\prime \prime \prime}-\right.$ $\left.\mathrm{N}\left(\mathrm{CH}_{3}\right)_{2}\right), 1.30\left(\mathrm{~d}, J=6.5 \mathrm{~Hz}, 3 \mathrm{H}, 15-\mathrm{CH}_{3}\right), 1.27$ (d, $J=6.5$ $\left.\mathrm{Hz}, 3 \mathrm{H}, 5^{\prime \prime \prime}-\mathrm{CH}_{3}\right), 1.18$ (d, $\left.J=6.5 \mathrm{~Hz}, 3 \mathrm{H}, 5^{\prime}-\mathrm{CH}_{3}\right), 1.16$ (s, $\left.3 \mathrm{H}, 3 "-\mathrm{CH}_{3}\right), 1.13$ (d, J=6.5 Hz, 3H, 5"-CH $), 0.96$ (d, $\left.J=6.5 \mathrm{~Hz}, 3 \mathrm{H}, 8-\mathrm{CH}_{3}\right), 0.88$ (m, 1H, H-7).

\section{4"-O-Benzoyl-4"'- $N$-demethylspiramycin I (10)}

Title compound $\mathbf{1 0}$ was prepared from compound $\mathbf{6}$ by Fmoc deprotection followed by methanolysis (58\%, 2 steps).

HR-MS (FAB) (matrix; $m$-NBA): calcd. for $\mathrm{C}_{98} \mathrm{H}_{149} \mathrm{~N}_{4} \mathrm{O}_{28}: 1830.0358[2 \mathrm{M}+\mathrm{H}]$, found: $m / z 1830.0358$ $[\mathrm{M}+\mathrm{H}]^{+}$.

IR $v_{\max }(\mathrm{KBr}) \mathrm{cm}^{-1}: 3490,2935,1733$.

${ }^{1} \mathrm{H}-\mathrm{NMR}\left(270 \mathrm{MHz}, \mathrm{CDCl}_{3}\right.$, partial data) $\delta(\mathrm{ppm}): 9.80$ (s, 1H, H-18), $8.11 \sim 8.08\left(\mathrm{~m}, 2 \mathrm{H}, 4^{\prime \prime}-\mathrm{OCOPh}\right), 7.55(\mathrm{~m}$, 1H, 4"-OCOPh), 7.47 7.42 (m, 2H, 4"-OCOPh), 6.23 (dd, $J=16.2,10.5 \mathrm{~Hz}, 1 \mathrm{H}, \mathrm{H}-11), 5.96$ (dd, $J=14.4,10.5 \mathrm{~Hz}$, 1H, H-12), 5.65 (dd, $J=15.1,9.6 \mathrm{~Hz}, 1 \mathrm{H}, \mathrm{H}-10), 5.53$ (ddd, $J=15.0,11.0,3.9 \mathrm{~Hz}, 1 \mathrm{H}, \mathrm{H}-13), 5.27$ (m, 1H, H-15), 5.11 (d, =3.2 Hz, 1H, H-1"), 4.98 (dd, $J=10.4,7.6 \mathrm{~Hz}, 1 \mathrm{H}, \mathrm{H}-2^{\prime}$ ), 4.81 (d, $\left.J=10.3 \mathrm{~Hz}, 1 \mathrm{H}, \mathrm{H}-4^{\prime \prime}\right), 4.72$ (d, $J=7.6 \mathrm{~Hz}, 1 \mathrm{H}, \mathrm{H}-$ $\left.1^{\prime}\right), 4.51$ (dq, $\left.J=10.1,6.5 \mathrm{~Hz}, 1 \mathrm{H}, \mathrm{H}-5^{\prime \prime}\right), 4.34$ (m, 1H, H-1"' ), 4.10 (m, 1H, H-5), 4.02 (dd, $J=9.6,3.8 \mathrm{~Hz}, 1 \mathrm{H}, \mathrm{H}-9)$, 3.78 (d, $J=11.1 \mathrm{~Hz}, 1 \mathrm{H}, \mathrm{H}-3), 3.45$ (s, 3H, 4-OCH $), 3.40$ (dq, $\left.J=9.5,6.2 \mathrm{~Hz}, 1 \mathrm{H}, \mathrm{H}-5^{\prime \prime \prime}\right), 3.30$ (m, 2H, H-4', H-5'), $2.98(\mathrm{~d}, J=9.0 \mathrm{~Hz}, 1 \mathrm{H}, \mathrm{H}-4), 2.43\left(\mathrm{~s}, 6 \mathrm{H}, 3^{\prime}-\mathrm{N}\left(\mathrm{CH}_{3}\right)_{2}\right), 2.20$ (s, 3H, 4"'-NHCH 3$), 1.28\left(\mathrm{~d}, J=6.2 \mathrm{~Hz}, 3 \mathrm{H}, 15-\mathrm{CH}_{3}\right), 1.22$ (d, $\left.J=6.5 \mathrm{~Hz}, 3 \mathrm{H}, 5^{\prime}-\mathrm{CH}_{3}\right), 1.21$ (s, 3H, 3"-CH3), 1.18 (d, $\left.J=6.5 \mathrm{~Hz}, 3 \mathrm{H}, \mathrm{C}^{\prime \prime}-\mathrm{CH}_{3}\right), 1.14\left(\mathrm{~d}, J=6.5 \mathrm{~Hz}, 3 \mathrm{H}, 5^{\prime \prime \prime}-\mathrm{CH}_{3}\right.$ ), 0.95 (d, $\left.J=6.5 \mathrm{~Hz}, 3 \mathrm{H}, 8-\mathrm{CH}_{3}\right), 0.87$ (m, 1H, H-7).

Acknowledgement This work was supported by a grant from the 21st Century COE Program, Ministry of Education, Culture,
Sports, Science and Technology. We also thank Ms. A. Nakagawa, Ms. C. Sakabe, and Ms. N. Sato (School of Pharmaceutical Sciences, Kitasato University) for the various instrumental analysis.

\section{References}

1. Pinnert-Sindico S, Ninet L, Preud'homme J, Cosar C. A new antibiotic-spiramycin. Antibiotics Ann. 1954/1955: 724-727 (1955)

2. Sano $\mathrm{H}$, Inoue $\mathrm{M}$, Yamashita $\mathrm{K}$, Okachi R, Ōmura S. Chemical modification of spiramycins. I. Synthesis of the acetal derivatives of neospiramycin I. J Antibiot 36: 13361344 (1983)

3. Sano H, Inoue M, Ōmura S. Chemical modification of spiramycins. II. Synthesis and antimicrobial activity of 4'deoxy derivatives of neospiramycin I and their 12-(Z)isomers. J. Antibiot. 37: 738-749 (1984)

4. Sano H, Sunazuka T, Tanaka H, Yamashita K, Okachi R, Ōmura S. Chemical modification of spiramycins. III. Synthesis and antibacterial activities of 4"-sulfonates and 4"alkylethers of spiramycin I. J Antibiot 37: 750-759 (1984)

5. Sano H, Sunazuka T, Tanaka H, Yamashita K, Okachi R, Ōmura S. Chemical modification of spiramycins. IV. Synthesis and in vitro and in vivo activities of 3 ",4"diacylates and 3,3",4"-triacylates of spiramycin I. J Antibiot 37: 760-772 (1984)

6. Sano H, Tanaka H, Yamashita K, Okachi R, Ōmura S. Chemical modification of spiramycins. V. Synthesis and antibacterial activity of $3^{\prime}$ - or $4^{\prime \prime \prime}$-de- $N$-methylspiramycin I and their $N$-substituted derivatives. J Antibiot 38: 186-196 (1985)

7. Sano H, Sunazuka T, Tanaka H, Yamashita K, Okachi R, Ömura S. Chemical modification of spiramycins. VI. Synthesis and antibacterial activities of 3,3"-di-O-acyl-4"-Osulfonyl and 3,3"-di-O-acyl-4"-O-alkyl derivatives of spiramycin I. J Antibiot 38: 1350-1358 (1985)

8. Yoshida K. Syntheses of new macrolides and the structureactivity relationships. $\mathrm{Ph}$. D. Thesis, Kitasato university (2005)

9. Hirose T, Sunazuka T, Noguchi Y, Yamaguchi Y, Hanaki H, Sharpless KB, Ōmura S. Rapid 'SAR' via click chemistry: an alkyne-bearing spiramycin is fused with diverse azides to yield new triazole-antibacterial candidates. Heterocycles 69: 55-61 (2006) 
10. Debono M, Willard KE, Kirst HA, Wind JA, Crouse GD, Tao EV, Vicenzi JT, Counter FT, Ott JL, Ose EE, Ōmura S. Synthesis and antimicrobial evaluation of 20-deoxo-20-(3,5- dimetylpiperidine-1-yl)desmicosin (Tilmicosin, EL-870. and related cyclic amino derivatives. J Antibiot 42: 12531267 (1989). 